\title{
Molecular gas in the Andromeda galaxy
}

\author{
Ch. Nieten ${ }^{1}$, N. Neininger ${ }^{1,2,3}$, M. Guélin ${ }^{3}$, H. Ungerechts ${ }^{4}$, R. Lucas ${ }^{3}$, E. M. Berkhuijsen ${ }^{1}$, \\ R. Beck ${ }^{1}$, and R. Wielebinski ${ }^{1}$ \\ 1 Max-Planck-Institut für Radioastronomie, Auf dem Hügel 69, 53121 Bonn, Germany \\ e-mail: c.nieten@zeiss.de; NNeininger@kpmg.com \\ 2 Radioastronomisches Institut der Universität Bonn, Auf dem Hügel 71, 53121 Bonn, Germany \\ 3 Institut de Radioastronomie Millimétrique, 300 rue de la piscine, 38406 St. Martin d' Hères, France \\ 4 Instituto de Radioastronomía Milimétrica, Avenida Divina Pastora 7, 18012 Granada, Spain
} e-mail: guelin@iram.fr

Received 13 November 2003 / Accepted 5 December 2005

\begin{abstract}
Aims. We study the distribution of the molecular gas in the Andromeda galaxy (M31) and compare this with the distributions of the atomic gas and the emission from cold dust at $\lambda 175 \mu \mathrm{m}$.

Methods. We obtained a new ${ }^{12} \mathrm{CO}(J=1-0)$-line survey of the Andromeda galaxy with the highest resolution to date (23", or $85 \mathrm{pc}$ along the major axis), observed $\mathrm{On}$-the-Fly with the IRAM 30-m telescope. We fully sampled an area of $2^{\circ} \times 0.5$ with a velocity resolution of $2.6 \mathrm{~km} \mathrm{~s}^{-1}$. In several selected regions we also observed the ${ }^{12} \mathrm{CO}(2-1)$-line.

Results. Emission from the ${ }^{12} \mathrm{CO}(1-0)$ line was detected from galactocentric radius $R=3 \mathrm{kpc}$ to $R=16 \mathrm{kpc}$ with a maximum in intensity at $R \sim 10 \mathrm{kpc}$. The molecular gas traced by the (velocity-integrated) (1-0)-line intensity is concentrated in narrow arm-like filaments, which often coincide with the dark dust lanes visible at optical wavelengths. Between $R=4 \mathrm{kpc}$ and $R=12 \mathrm{kpc}$ the brightest CO filaments define a two-armed spiral pattern that is described well by two logarithmic spirals with a pitch angle of $7^{\circ}-8^{\circ}$. The arm-interarm brightness ratio averaged over a length of $15 \mathrm{kpc}$ along the western arms reaches about 20 compared to 4 for $\mathrm{H} \mathrm{I}$ at an angular resolution of $45^{\prime \prime}$. For a constant conversion factor $X_{\mathrm{CO}}$, the molecular fraction of the neutral gas is enhanced in the spiral arms and decreases radially from 0.6 on the inner arms to 0.3 on the arms at $R \simeq 10 \mathrm{kpc}$. The apparent gas-to-dust ratios $N(\mathrm{H} \mathrm{I}) / I_{175}$ and $\left(N(\mathrm{H} \mathrm{I})+2 N\left(\mathrm{H}_{2}\right)\right) / I_{175}$ increase by a factor of $\sim 20$ between the centre and $R \simeq 14 \mathrm{kpc}$, whereas the ratio $2 N\left(\mathrm{H}_{2}\right) / I_{175}$ only increases by a factor of 4 .

Conclusions. Either the atomic and total gas-to-dust ratios increase by a factor of $\sim 20$ or the dust becomes colder towards larger radii. A strong variation of $X_{\mathrm{CO}}$ with radius seems unlikely. The observed gradients affect the cross-correlations between gas and dust. In the radial range $R=8-14 \mathrm{kpc}$ total gas and cold dust are well correlated; molecular gas correlates better with cold dust than atomic gas. The mass of the molecular gas in M 31 within a radius of $18 \mathrm{kpc}$ is $M\left(\mathrm{H}_{2}\right)=3.6 \times 10^{8} M_{\odot}$ at the adopted distance of $780 \mathrm{kpc}$. This is $7 \%$ of the total neutral gas mass in M 31 .
\end{abstract}

Key words. ISM: molecules - galaxies: individual: M 31 - galaxies: ISM - galaxies: spiral - radio lines: galaxies

\section{Introduction}

Star formation and spiral structure in galaxies require the coupling of processes operating on linear scales so different that they are hard to study in a single galaxy. The small structures are difficult to observe in external galaxies, whereas large structures are hard to see in the Milky Way due to distance ambiguities. Singledish telescopes were used to survey CO in galaxies (e.g. Nakano et al. 1987; Braine et al. 1993; Young et al. 1995) but with limited angular resolution. Molecular spiral arms were barely resolved in these surveys even in the nearest galaxies (e.g. Koper et al. 1991; Garcia-Burillo et al. 1993; Loinard et al. 1996; Heyer et al. 2004). Only in the Magellanic Clouds did the single-dish surveys resolve giant molecular clouds (Israel et al. 1993). More recently mm-wave interferometer surveys like the BIMA SONG (Regan et al. 2001) gave vastly improved data on nearby galaxies like M 51, resolving molecular arms into cloud complexes. This instrument was also used for an all-disk survey of M 33, about ten times closer to us than M 51, in which individual molecular clouds are recognized (Engargiola et al. 2003). The IRAM Plateau de Bure interferometer has resolved molecular clouds in
M31 into components (Neininger et al. 2000a) enabling close comparisons with molecular clouds in the Milky Way.

The nearest large spiral is the Andromeda Nebula, M31. Its distance of $D=0.78 \pm 0.04 \mathrm{Mpc}$ (Stanek \& Garnavich 1998) ranks among the best known for any galactic or extragalactic nebula; the accuracy of this distance allows us to derive accurate luminosities and masses. At this distance, $1^{\prime}$ along the major axis corresponds to $227 \pm 12$ pc. The large inclination of M 31, $i=77^{\circ} .5$, degrades the resolution along the minor axis by a factor of 4.6, but has the advantage of yielding accurate in-plane velocities. The proximity of M31 gives us the chance to see many details of the distribution and kinematics of the gas, as well as the relation of the gas to the spiral structure and to star formation.

The contents of stars, dust, and atomic gas in M 31 are well known. The whole galaxy has been mapped in the $21 \mathrm{~cm}$ line of H I with $24^{\prime \prime} \times 36^{\prime \prime}$ resolution by Brinks \& Shane (1984, hereafter $\mathrm{B} \& \mathrm{~S})$ and its northeastern half with $10^{\prime \prime}$ resolution by Braun (1990). It has been entirely mapped in the mid and far infrared by the IRAS, ISO, and Spitzer satellites (see Haas et al. 1998 and Schmidtobreick et al. 2000 for the ISOPHOT map at $175 \mu \mathrm{m}$, and Gordon et al. 2004 for the MIPS maps at $20 \mu \mathrm{m}, 60 \mu \mathrm{m}$ 
and $160 \mu \mathrm{m})$. Furthermore, M 31 was partially mapped with ISOCAM (5.1-16.5 $\mu \mathrm{m}$ ) at $6^{\prime \prime}$ resolution (see e.g. Pagani et al. 1999). Comparisons of the emission in different wavelength ranges - like UV, optical, HI, FIR $(160 \mu \mathrm{m}$ and $175 \mu \mathrm{m})$ and radio continuum emissions - have also been reported (Loinard et al. 1999; Pagani et al. 1999; Keel 2000; Lequeux 2000; Nieten et al. 2000; Berkhuijsen et al. 2000; Gordon et al. 2004).

So far, the situation was not as favourable for the molecular gas. Prior to ours, the only complete CO survey of M 31 was made with a 1.2-m diameter telescope and had a resolution of 8.7 (Koper et al. 1991; Dame et al. 1993). More recently, a survey of the southwestern half, made at an angular resolution of $1^{\prime}$ with the FCRAO 14-m telescope, was published by Loinard et al. (1996, 1999). The latter authors (1999, their Table 2) give a nearly complete overview of previous CO observations of M31. Loinard et al. (1999) and Heyer et al. (2000) found many similarities, but also clear differences, between properties of the molecular gas in M31 and those in the Milky Way.

Our survey, made with the IRAM 30-m telescope in the ${ }^{12} \mathrm{CO}(J=1-0)$ line, has a resolution of 23 " corresponding to $85 \mathrm{pc}$ along the major axis. It is much more sensitive than the previous surveys and detects all clouds with $\int T_{\mathrm{mb}} \mathrm{dv} \gtrsim$ $1 \mathrm{~K} \mathrm{~km} \mathrm{~s}^{-1}$ (=3× rms noise). In this article we present the $\mathrm{CO}$ distribution in the bright disk of the galaxy. We derive some important basic results using simple assumptions, e.g., a constant conversion factor $X_{\mathrm{CO}}$ from CO intensity to molecular column density. We discuss the spiral-arm structure of the neutral gas and the arm-interam brightness contrast in Sect. 3. In addition to the ${ }^{12} \mathrm{CO}(J=1-0)$ line, we observed several selected areas covering bright arm segments in the ${ }^{12} \mathrm{CO}(J=2-1)$ line with high sensitivity; we discuss the line ratios in Sect. 3.3. In a previous publication, based on one third of the present data (Neininger et al. 1998), we reported a tight correlation between the CO sources and the dark dust lanes. In Sect. 4 we return to this point and compare the CO distribution with those of H I, FIR $(175 \mu \mathrm{m})$ and $\lambda 20 \mathrm{~cm}$ radio continuum. Radial profiles of the various constituents are discussed in Sect. 4.1 and correlations between CO, H I and FIR $(175 \mu \mathrm{m})$ in Sect. 4.2. In Sect. 4.3 we derive the molecular and total gas mass. The CO velocity field is described in Sect. 5. Our results are summarized in Sect. 6. Preliminary reports on this survey are given by Guélin et al. (2000), Neininger et al. $(1998,2000 b)$ and Nieten et al. (2000).

\section{Observations}

Our survey was carried out with the IRAM 30-m telescope between November 1995 and August 2001. The observations were made On-the-Fly in two steps: in a first step a field typically $18^{\prime} \times 18^{\prime}$ in size was scanned back and forth in the direction parallel to M31's minor axis, $Y$, at a speed of $4^{\prime \prime} \mathrm{s}^{-1}$. The successive scans were spaced by $9^{\prime \prime}$ in the orthogonal $X$ direction. At the beginning and at the end of each scan, a reference position, located $30^{\prime}$ or $45^{\prime}$ away from the major axis ${ }^{1}$ and free of $\mathrm{CO}$ or H I emission, was observed for $30 \mathrm{~s}$. Every 1-2 h the telescope pointing was checked on planets and nearby quasars. A second reference position, located within M31 and showing strong CO emission, was observed for calibration purposes (see below). The telescope focus was checked several times a day, in

1 Throughout this paper we use the M31-fixed coordinate system of Baade \& Arp (1964): centre coordinates RA $=0^{\mathrm{h}} 40^{\mathrm{m}} 00^{\mathrm{s}} 3$, Dec $=$ $41^{\circ} 00^{\prime} 03^{\prime \prime}$ (1950.0) (Dressel \& Condon 1976), $X$ along the major axis $\left(\mathrm{PA}=37^{\circ} .7\right)$, positive to the NE, $Y$ along the minor axis, positive to the SE. All velocities are in the LSR frame. particular after sunrise and sunset. In a second step the observations were repeated by scanning the same field in the orthogonal direction, parallel to the major axis. The data recorded by the backends were read every second of time, so that the data cube obtained by combining the two orthogonal maps was fully sampled on a $9^{\prime \prime}$ grid.

The reduction procedure was described in some detail by Neininger et al. (2000b). After calibration (see below), subtraction of the off-source reference spectrum and a baseline for individual spectra in a map, two orthogonal maps were combined using "basket-weaving", the de-striping technique of Emerson \& Gräve (1988). This code was adapted to work on two orthogonal channel maps before averaging them (Hoernes 1997). Examples of the technique are shown in Neininger et al. (2000b), Hoernes (1997) and Emerson \& Gräve (1988). In this process, the CO map was smoothed from $21^{\prime \prime}$ to $23^{\prime \prime}$ FWHM.

The maps shown in Fig. 1 are the combination of 12 individual fields, as listed in Table 1. Each field is fully sampled and all fields together contain nearly 1.7 million spectra (before gridding) obtained in about $500 \mathrm{~h}$ of effective observing time. We present individual spectra for selected regions in Fig. 2.

When observing the $\mathrm{CO}(1-0)$ line, the $30-\mathrm{m}$ telescope allows simultaneous observations of two polarizations of the (2-1) line. The (2-1) line, however, is weaker than the (1-0) line, and the integration time per beam is about $4 \times$ smaller because the beam area is $4 \times$ smaller. Moreover, the receivers are noisier and the sky opacity is higher at $230 \mathrm{GHz}(2-1)$ than at $115 \mathrm{GHz}(1-0)$. This made it difficult to detect the (2-1)-line emission in our survey, except for the brightest clouds. In order to improve the signal-to-noise ratio and the sampling for $(2-1)$, we re-observed several rectangular regions, $3^{\prime}-4^{\prime}$ wide by $3^{\prime}-12^{\prime}$ long, with half the scanning velocity and twice the sampling, when the zenith opacity at $230 \mathrm{GHz}$ was favourably low $(\leq 0.2)$. We discuss some general results of these observations in Sect. 3.3. In order to confirm the reliability of the OTF method, to integrate some emission-free positions to a lower noise level and to check several apparent discrepancies with previous $\mathrm{CO}$ observations, we re-observed about 200 positions, located inside as well as outside the arms, in the position-switching mode with integration times of 10-30 $\mathrm{min}$. Some of these results are used in Sect. $3.2^{2}$.

We used two SIS receivers with orthogonal polarizations to observe the (1-0) line and a similar system to observe the $(2-1)$ line. The receiver temperatures in the standard reference plane (before the polarization splitter) were close to $90 \mathrm{~K}$ (SSB) at $115 \mathrm{GHz}$ at the beginning of our survey (fields $1-3$, see Table 1) and close to $50 \mathrm{~K}$ at the end (fields 8-11). After addition of the atmospheric contribution, the system temperature was between 200 and $400 \mathrm{~K}$ at both $115 \mathrm{GHz}$ and at $230 \mathrm{GHz}$. The backends consisted of two $512 \times 1 \mathrm{MHz}$ filterbanks at $115 \mathrm{GHz}$ and of 2 autocorrelators with resolutions of $0.8 \mathrm{MHz}$ and total bandwidth of $320 \mathrm{MHz}$ at $230 \mathrm{GHz}$. A channel width of $1 \mathrm{MHz}$ corresponds to a velocity resolution of $2.6 \mathrm{~km} \mathrm{~s}^{-1}$ for the (1-0) line and of $1.3 \mathrm{~km} \mathrm{~s}^{-1}$ for the (2-1) line.

The standard calibration at the $30-\mathrm{m}$ telescope is equivalent to the chopper-wheel calibration method for observations at millimeter wavelengths; it gives the antenna temperature $T_{\mathrm{A}}^{*}$, corrected for atmospheric losses and forward efficiency, $F_{\text {eff }}$ (for details see Downes 1989). The main-beam brightness temperature, $T_{\mathrm{mb}}$, can then be calculated from

$T_{\mathrm{mb}}=F_{\mathrm{eff}} / B_{\mathrm{eff}} T_{\mathrm{A}}^{*}$,

${ }^{2}$ The full results of the position-switched observations will be presented elsewhere. 

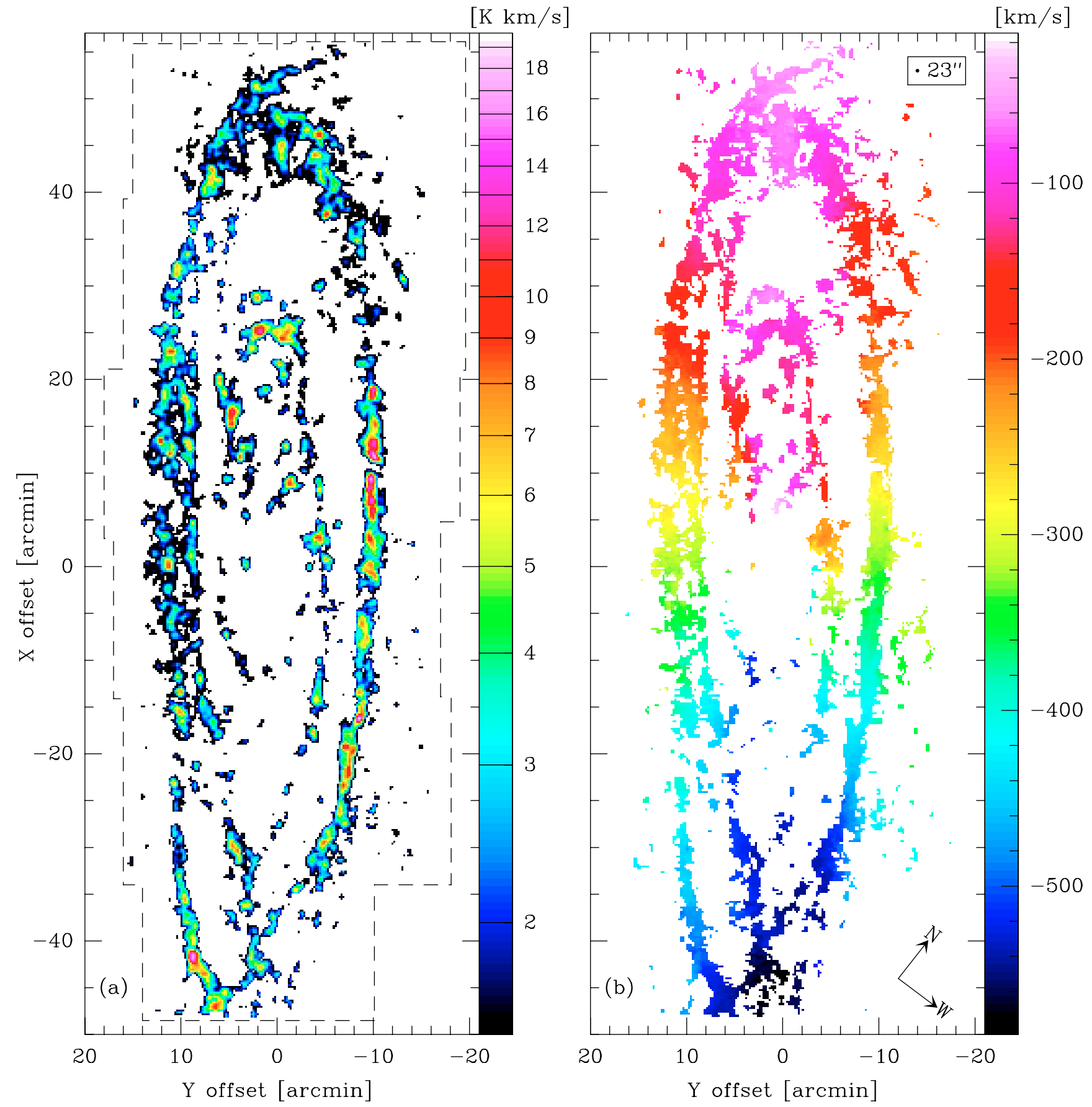

Fig. 1. a) The velocity-integrated intensity distribution of the ${ }^{12} \mathrm{CO}(1-0)$ spectrum, $I_{1-0}=\int T_{\mathrm{mb}}\left(\mathrm{CO}_{1-0}\right)$ dv, observed with the IRAM $30-\mathrm{m}$ telescope. The $X$ and $Y$ coordinates are taken along the major and minor axis, respectively; the position angle and centre position are taken from Dressel \& Condon (1976 - see footnote 1). The dashed line marks the border of the area surveyed, which is about one degree squared. The colour scale starts at $1.5 \mathrm{~K} \mathrm{~km} \mathrm{~s}^{-1}$. Most structures visible in the map, including weak ones, correspond to line profiles detected with signal-to-noise ratios $\geq 4$. The mean rms noise in the integrated intensities is $0.35 \mathrm{~K} \mathrm{~km} \mathrm{~s}^{-1}$, but the noise varies across the map (see Table 1). b) The velocity field (first moment) as traced by the $\mathrm{CO}$ emission. Velocities are $L S R$-values.

where $B_{\text {eff }}$ is the main-beam efficiency. Over the years of this project, $F_{\text {eff }}$ and in particular $B_{\text {eff }}$ improved significantly due to upgrades of the $30-\mathrm{m}$ telescope, the most important of which occurred in July 1997. We used the standard values for these efficiencies for the last quarter of 1997 derived by the observatory staff, for $F_{\text {eff }}$ from antenna tippings and for $B_{\text {eff }}$ from observations of Mars and Uranus: $F_{\text {eff }} / B_{\text {eff }}=0.92 / 0.80$ at $115 \mathrm{GHz}$ and $0.80 / 0.50$ at $230 \mathrm{GHz}$. Day-to-day variations were monitored by observing two reference positions: $X, Y=-30$ '.18, 4.26 in the south and $X, Y=18$ '.80, 1'.90 in the north. Normalization of the line integrals at these positions to the same values ensured the same calibration parameters for the entire survey. The uncertainty in the final $T_{\mathrm{mb}}$ scales is about $15 \%$.

A corollary of the high beam efficiency of the 30-m telescope at $115 \mathrm{GHz}$ is the low error beam. Greve et al. (1998) found that the far-beam pattern can be described by 3 error beams with half-power widths of $5^{\prime}, 7^{\prime}$ and $80^{\prime}$, respectively. The first two could pick up signals from regions with similar radial velocities as observed in the main beam. As they contributed only a few percent to the main-beam signals, they hardly affected the 
Table 1. Data on observed fields of M31.

\begin{tabular}{|c|c|c|c|c|c|c|}
\hline \multirow{2}{*}{$\begin{array}{r}\text { Field } \\
\#\end{array}$} & \multirow{2}{*}{$\begin{array}{l}\text { Quadrant } \\
\text { in M31 }\end{array}$} & \multirow{2}{*}{$\begin{array}{c}X \text {-extent } \\
\left({ }^{\prime}\right) \\
\end{array}$} & \multirow{2}{*}{$\begin{array}{c}\text {-extent } \\
\left({ }^{\prime}\right) \\
\end{array}$} & \multirow{2}{*}{$\begin{array}{r}\text { Number } \\
\text { of spectra }\end{array}$} & \multicolumn{2}{|c|}{ Mean rms noise } \\
\hline & & & & & $\begin{array}{c}\text { in } I_{1-0} \\
\left(\mathrm{~K} \mathrm{~km} \mathrm{~s}^{-1}\right)\end{array}$ & $\begin{array}{l}\text { per channel } \\
(\mathrm{mK})^{1)}\end{array}$ \\
\hline 1 & $\bar{S}$ & $-48.5 \ldots-33.3$ & $-10.1 \ldots 14.0$ & 162980 & 0.45 & 34 \\
\hline 2 & SW & $-34.0 \ldots-13.9$ & $-18.1 \ldots-2.0$ & 225122 & 0.38 & 37 \\
\hline 3 & SE & $-34.0 \ldots-13.9$ & $2.0 \ldots 16.1$ & 115765 & 0.35 & 34 \\
\hline 4 & SW & $-14.2 \ldots \quad 5.1$ & $-17.0 \ldots-3.0$ & 128027 & 0.44 & 33 \\
\hline 5 & NE & $21.0 \ldots \quad 39.3$ & $-2.0 \ldots 16.1$ & 204190 & 0.46 & 32 \\
\hline 6 & NW & $21.0 \ldots \quad 39.1$ & $-19.6 \ldots-1.5$ & 152630 & 0.25 & 22 \\
\hline 7 & NE & $39.0 \ldots \quad 55.9$ & $-2.0 \ldots 15.1$ & 160045 & 0.27 & 21 \\
\hline 8 & NW & $38.9 \ldots \quad 56.1$ & $-19.6 \ldots-1.5$ & 117198 & 0.29 & 27 \\
\hline 9 & $\mathrm{NE}$ & $3.0 \ldots$ & $-2.0 \ldots 18.0$ & 164760 & 0.32 & 25 \\
\hline 10 & SE & $-14.1 \ldots$ & $-3.0 \ldots 17.0$ & 128534 & 0.27 & 27 \\
\hline 11 & NW & $4.8 \ldots$ & $-19.0 \ldots-1.0$ & 128156 & 0.33 & 24 \\
\hline 12 & NW & $2.8 \ldots \quad 5.1$ & $-3.0 \ldots-1.8$ & 666 & 0.36 & 26 \\
\hline & $\mathrm{NE}$ & $X>0$ & $Y>0$ & 528995 & $0.36^{2)}$ & $26^{2)}$ \\
\hline & NW & $X>0$ & $Y<0$ & 398650 & 0.29 & 24 \\
\hline & SE & $X<0$ & $Y>0$ & 339371 & 0.35 & 31 \\
\hline & SW & $X<0$ & $Y<0$ & 421057 & 0.41 & 35 \\
\hline & Total & 3512 square arc & inutes & 1688073 & 0.35 & 29 \\
\hline
\end{tabular}

1) Temperatures are on $T_{\mathrm{mb}}$-scale, where $T_{\mathrm{mb}}=1.15 T_{\mathrm{A}}^{*} .{ }^{2)}$ Weighted means.

observed spectra. The contribution of the third error beam, which is larger than the disk of M 31, was negligible.

The effective integration time per $23^{\prime \prime}$ beam for the large ${ }^{12} \mathrm{CO}(1-0)$ map was $64 \mathrm{~s}$, yielding a rms noise of $\simeq 33 \mathrm{mK}$ per $1 \mathrm{MHz}$ channel $\left(T_{\mathrm{mb}}\right.$ scale) in the southern fields $(1-4)$ and $\simeq 25 \mathrm{mK}$ per $1 \mathrm{MHz}$ in the northern ones (see Table 1 ). The corresponding values for the small maps are $21^{\prime \prime}, 173 \mathrm{~s}$ and $15 \mathrm{mK}$ for the (1-0) line, and $12^{\prime \prime}, 57 \mathrm{~s}$ and $35 \mathrm{mK}$ per $1 \mathrm{MHz}$ channel for the (2-1) line.

The distribution of velocity-integrated $\mathrm{CO}$-line intensities, $I_{1-0}=\int T_{\mathrm{mb}}\left(\mathrm{CO}_{1-0}\right) \mathrm{d} v$, is shown in Fig. 1a. The rms noise in the velocity-integrated emission varies between the fields, but is typically about $0.38 \mathrm{~K} \mathrm{~km} \mathrm{~s}^{-1}$ in the southern part and about $0.33 \mathrm{~K} \mathrm{~km} \mathrm{~s}^{-1}$ in the northern half of the survey or $0.35 \pm$ $0.10 \mathrm{~K} \mathrm{~km} \mathrm{~s}^{-1}$ for the total map (see Table 1). We note that the sensitivity of our survey to point-like and extended sources exceeds that of Loinard et al. (1999) by factors $>8$ and $>1.5$, respectively.

\section{The $\mathrm{CO}$ brightness distribution}

The survey of the Andromeda galaxy presented in this paper is the largest and most detailed molecular-line survey ever made of an extragalactic object. Most of the emission in Fig. 1 appears concentrated on radii between 3 and $12 \mathrm{kpc}$ and occurs as long and narrow filaments that strongly suggest a spiral arm structure. In addition, we see a number of scattered $\mathrm{CO}$ clouds of weak intensity between the spiral arms; sometimes these form bridges. The inner arms at radii near $5 \mathrm{kpc}$ are remarkably bright, especially in the NE half of the galaxy.

Close to the centre of M31, CO emission is very weak. Melchior et al. (2000) have found $\mathrm{CO}$ emission of about $T_{\mathrm{A}}^{*}=$ $20 \mathrm{mK}$ in a dark-cloud complex located at a distance of $350 \mathrm{pc}$ from the centre using position switching and long integrations. The integration time per beam was about $6 \mathrm{~h}$ and the rms noise $2 \mathrm{mK}$ per $3 \mathrm{~km} \mathrm{~s}^{-1}$-wide channel, which is much better than the noise in the present survey.

Only a few scattered clouds are visible at large distances from the centre. The most distant cloud in this survey was found at a deprojected radius of $19.4 \mathrm{kpc}$. The spectrum of this cloud is shown in Fig. 2a. The central velocity of the emission fits well to the velocity field of M31 (see Fig. 1b). The cloud is located in the outermost part of the spiral arm A-N modelled by Braun (1991) near several H II regions.

In the following subsections we model the spiral pattern and analyse the arm-interarm contrast.

\subsection{The structure of molecular spiral arms}

Although M 31 was classified at the beginning by Hubble (1929) as an $S b$ type spiral, attempts to draw its spiral pattern have mostly been inconclusive. For example, Baade (1963) used the young stars and Hodge (1979) the open star clusters as spiral arm tracers. These results were summarized by Hodge (1981b). Due to absorption of optical light by dust-rich lanes, the presence of a bright optical bulge and the lack of $\mathrm{H}$ I radio line emission in the inner disk, there was no consensus even on whether the arms are trailing or leading. More recently, Braun (1991) proposed a non-planar trailing two-armed spiral pattern with varying pitch angle on the basis of the $\mathrm{HI}$ interferometric data. His model accounts fairly well for the outer $\mathrm{H}$ I arms, but it does not trace the innermost structures. Based on their analysis of the $175 \mu \mathrm{m}$ dust emission, Haas et al. (1998) suggested that M31 may be closer to a ring galaxy than to a spiral.

The CO emission, which traces the dense molecular gas, is better suited to determining M31's spiral pattern because the $\mathrm{CO}$ arms are: (i) thinner than the $\mathrm{H}$ I arms; (ii) less patchy than the $\mathrm{H} \alpha$ arms; and (iii) not affected by absorption. As discussed below, the $\mathrm{CO}$ arm-interarm contrast is also much higher than in H I. Furthermore, the linear resolution of the CO survey is sufficient for distinguishing neighbouring arms even on the minor axis.

In order to determine the spiral pattern in an objective way, we decomposed the CO map into 170 individual "clouds" using the CLUMPFIND analysis program (Williams et al. 1994). CLUMPFIND provided the position of "clouds", deprojected them (assuming an inclination of the molecular disk of $i=77.5$ ) and transformed them into polar coordinates. The resulting data points, plotted in semi-logarithmic coordinates, were then leastsquare fitted with two straight lines representing two simple logarithmic spirals (see Figs. 3a and b). The fits led to very similar pitch angles for the two spirals, namely $\psi=8^{\circ} \pm 1^{\circ}$ and 

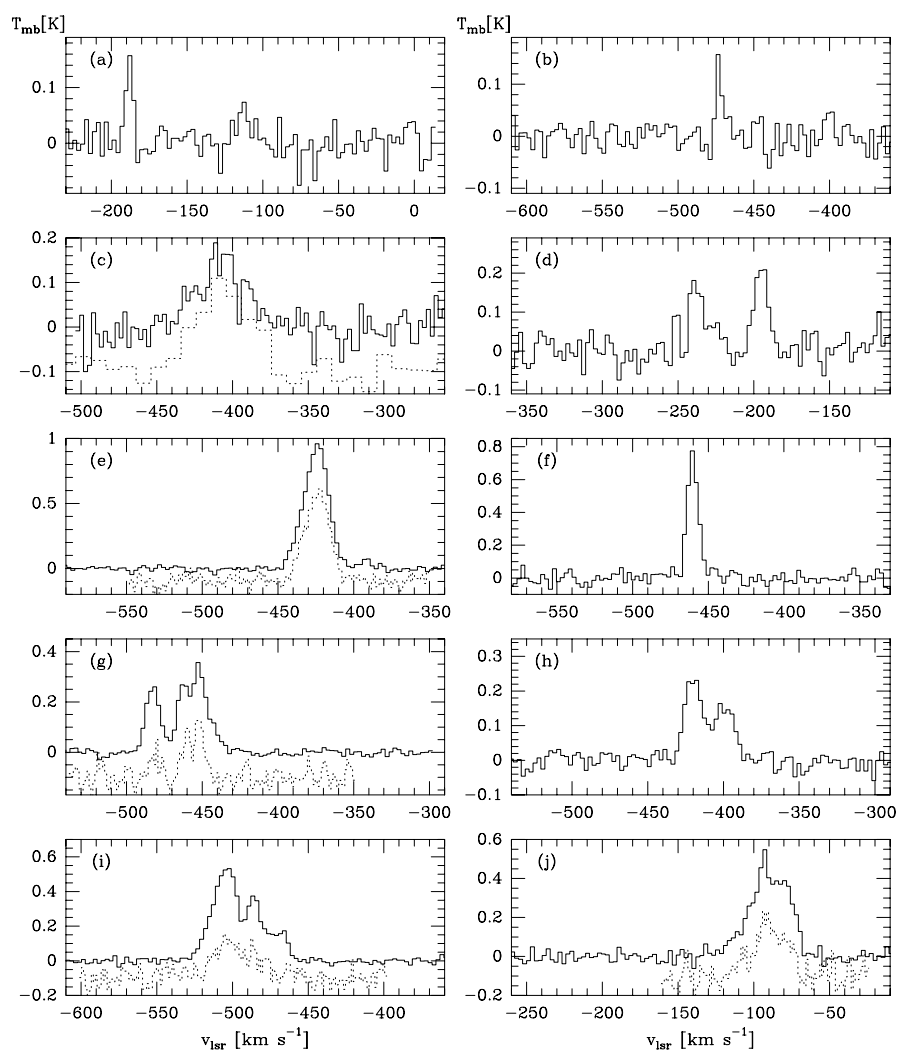

Fig. 2. Sample of spectra extracted from the data cube of the ${ }^{12} \mathrm{CO}(1-0)$ survey of $\mathrm{M} 31$ (full lines). At some positions the ${ }^{12} \mathrm{CO}(2-1)$ spectrum is also shown (dotted lines, shifted down by $0.1 \mathrm{~K}$ for clarity). The velocity resolution is $2.6 \mathrm{~km} \mathrm{~s}^{-1}$ for the $(1-0)$ line and $1.3 \mathrm{~km} \mathrm{~s}^{-1}$ for the (2-1) line. Velocities are in the LSR frame. a) Weak emission at $X, Y=46{ }^{\prime} 1,-15^{\prime} \cdot 6$ that corresponds to a deprojected distance from the centre of $19.4 \mathrm{kpc}$. b) Spectrum with linewidth $<5 \mathrm{~km} \mathrm{~s}^{-1}$ at $X, Y=$ $-46^{\prime} .08,9^{\prime} .35$. c) Broad line of about $40 \mathrm{~km} \mathrm{~s}^{-1}$ close to the minor axis at $X, Y=-0.33,-3.49$. The $(2-1)$ spectrum is smoothed to $9.9 \mathrm{~km} \mathrm{~s}^{-1}$. d) Two peaks separated by about $40 \mathrm{~km} \mathrm{~s}^{-1}$ at $X, Y=3.02,-4.97$. e) Emission from the dust cloud D 84 at $X, Y=-16.528,-8.728$; strongest emission is on the $10-\mathrm{kpc}$ arm. f) Emission at the position $X, Y=-17 ! 8,-3{ }^{\prime} 85$ near the dust cloud D 153. g) Emission with three velocity components near the dust cloud D 47 at $X, Y=-22 ! 48,-7 \cdot 53$. h) Double-peak emission from the inner arm, $X, Y=-7.57$, 5'.115. i) Emission with three components near the dust cloud D 39, $X, Y=$ $-41^{\prime} 9,8,54$. j) Emission at the position $X, Y=24.85,1$, .62 near the dust cloud D 615.

$7^{\circ} \pm 1^{\circ}$, and to a phase shift of roughly $200^{\circ}$. The derived pitch angles agree well with the value of $\psi=6.7$ for the mean of the 2 spiral arms observed in H I (Braun 1991) and with the mean value of $7^{\circ} .4$ for the optical spiral arms (Baade \& Arp 1964). The oscillations visible in Fig. $3 \mathrm{~b}$ indicate variations in pitch angle that could be partly due to variations in inclination angle observed by Braun (1991).

We note that these geometrical spiral fits required centre positions shifted to the NE with respect to the nucleus of M31, i.e. $X_{\mathrm{c}}, Y_{\mathrm{c}}=7: 7,0.4$ for the full-line spiral and $X_{\mathrm{c}}, Y_{\mathrm{c}}=5.8,2 ! 3$ for the dashed spiral (Figs. $3 \mathrm{a}$ and $\mathrm{b}$ ). Such a displacement is not surprising, as the bulge of M 31 is likely to host a bar (Stark \& Binney 1994), which makes it difficult to trace the spiral arms down to the nucleus. This displacement and the fact that on the minor axis the outer $\mathrm{CO}$ arms lie slightly outside the fitted spirals may also indicate that the molecular spiral arms have inclinations that differ from the adopted inclination of 77.5 of the main plane of M31, like the H I arms (Braun 1991). For a detailed comparison with the $\mathrm{H}$ I arms, a more complete analysis of the molecular arm structure should be made that also includes the velocity structure of the arms. This could be the subject of a future study.

For comparison, we tried to represent the $\mathrm{CO}$ emission at $R=8-12 \mathrm{kpc}$ by a circular ring, as suggested by Haas et al. (1998). The result of the fit is shown in Fig. 3c. Even at these radii the spiral pattern appears to be a better description of the $\mathrm{CO}$ distribution than a ring-like structure.

The derived spiral pattern is a good fit to the $\mathrm{CO}$ emission up to about $12 \mathrm{kpc}$ from the centre, except for the region of the giant stellar association NGC $206\left(X, Y=-40^{\prime},+22^{\prime}\right)$, the velocity of which strongly deviates from the normal rotation velocity. In particular, it accounts for the splitting of the $\mathrm{CO}$ emission along the eastern minor axis into two distinct arms. These arms are clearly seen in the $8 \mu \mathrm{m}$ emission from hot dust (MSX satellite map, Moshir et al. 1999; ISOCAM map of the central bulge of Willaime et al. 2001, see Fig. 7 below) and are also predicted by Braun's model although they are only marginally visible in H I and $\mathrm{H} \alpha$ emissions.

At radii $\gtrsim 12 \mathrm{kpc}$ the fitted spiral pattern starts to deviate from the observed spiral. There the $\mathrm{CO}$ emission becomes fainter and the filaments more difficult to trace. We note, however, that the ISO $175 \mu \mathrm{m}$ map of Haas et al. (1998) and the $\mathrm{H} \alpha$ image of Walterbos (2000) show a clear arm-like structure that seems to lie on the extension of the fitted spiral to the NE, far beyond our CO image.

\subsection{Arm-interarm contrast}

To derive the apparent arm width and the arm-interarm brightness intensity ratio, we divided M31's disk into 4 quadrants of approximately equal size. For each quadrant, we generated a family of spiral segments from a linear combination of the spirals that best fit the inner and outer $\mathrm{CO}$ arms. Figure 4a shows a sample of the spiral segments generated for the NW quadrant, superposed onto the CO map smoothed to a resolution of $45^{\prime \prime}$. We then calculated the average $\mathrm{CO}$ intensity along each spiral segment. Figure $4 \mathrm{~b}$ shows these averages for the 4 quadrants as functions of the spiral segment radii at their intersection with the major or minor axis. In this analysis, we discarded the highly perturbed region surrounding NGC 206 in the SW quadrant.

The CO peak-to-dip ratio reaches a maximum $>20$ in all quadrants, showing that the arm-interarm intensity contrast is high almost everywhere. The shallow dip at $R=11 \mathrm{kpc}$ in the SE quadrant reflects that the two brightest spiral arms $(R=9 \mathrm{kpc}$ and $12 \mathrm{kpc}$ ) are only partly resolved in this quadrant (see Fig. 1). The shoulders visible in Fig. 4 at $R=9 \mathrm{kpc}$ (NE quadrant) and at $10 \mathrm{kpc}$ (SW quadrant) come from two weak, nearly symmetrical arm-like substructures that are clearly visible in Fig. 1 (e.g. from $X, Y=27^{\prime}, 8^{\prime}$ to $X, Y=40^{\prime}, 2^{\prime}$ in the NE quadrant).

That $\mathrm{CO}$ emission is very low between the arms and outside these substructures is supported by the more than one hundred pointed observations we made in the interarm regions, in particular in the direction of several weak and extended interarm features that appear on the map of Loinard et al. (1999). Our observations failed to reveal any emission down to levels 3-5 times lower than the sensitivity of our OTF survey. Similarly, a strip of $8^{\prime}$ length centred on $X, Y=15^{\prime}, 6^{\prime}$, about half-way between the $5 \mathrm{kpc}$ and the $10 \mathrm{kpc}$ arms, did not show emission above $0.13 \mathrm{~K} \mathrm{~km} \mathrm{~s}^{-1}$ (1 s.d. at $23^{\prime \prime}$ resolution), which is 30 times below the average intensity along the corresponding segment at the crest of the $10 \mathrm{kpc}$ arm.

The apparent half-power width of the arms in Fig. $4 \mathrm{~b}$ is $1-2 \mathrm{kpc}$. This includes the true arm width within the plane of 

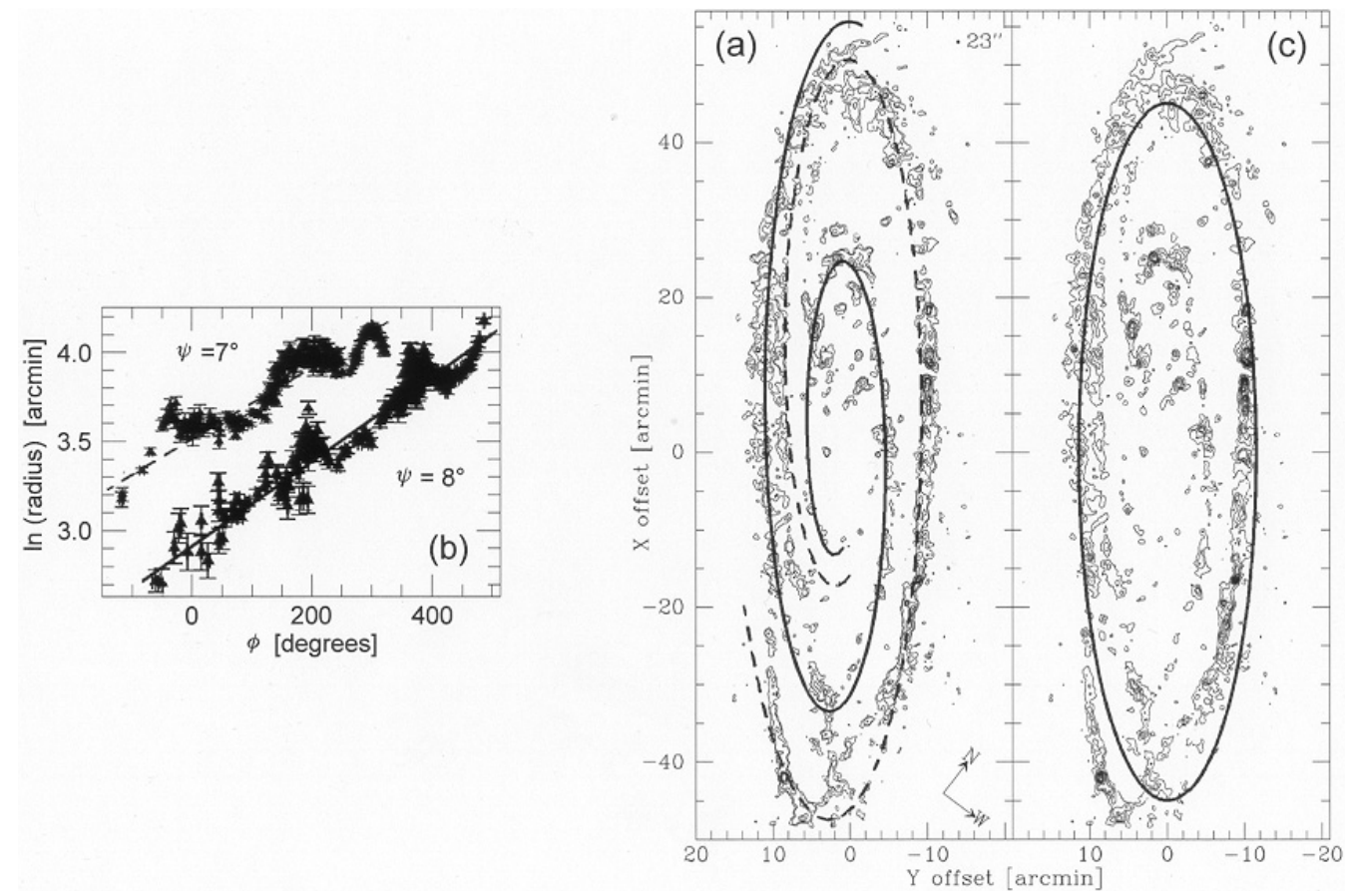

Fig. 3. Spiral structure in M31. a) Two logarithmic spirals (solid and dashed lines) fitted through the distribution of CO "clouds" in the radial range $R=4-12 \mathrm{kpc}$. They are overlayed onto the $\mathrm{CO}$ brightness distribution (contours). b) Least-square fits in the $\ln R-\phi$ plane yielding pitch angles of $8^{\circ}$ and $7^{\circ}$ for the solid and dashed spirals in a), respectively. The azimuthal angle $\phi$ is counted clockwise from the eastern minor axis in the plane of M31. c) Ring fitted to the distribution of "clouds" at $R=9-12 \mathrm{kpc}$ (solid line) superposed onto the CO-brightness distribution (contours).
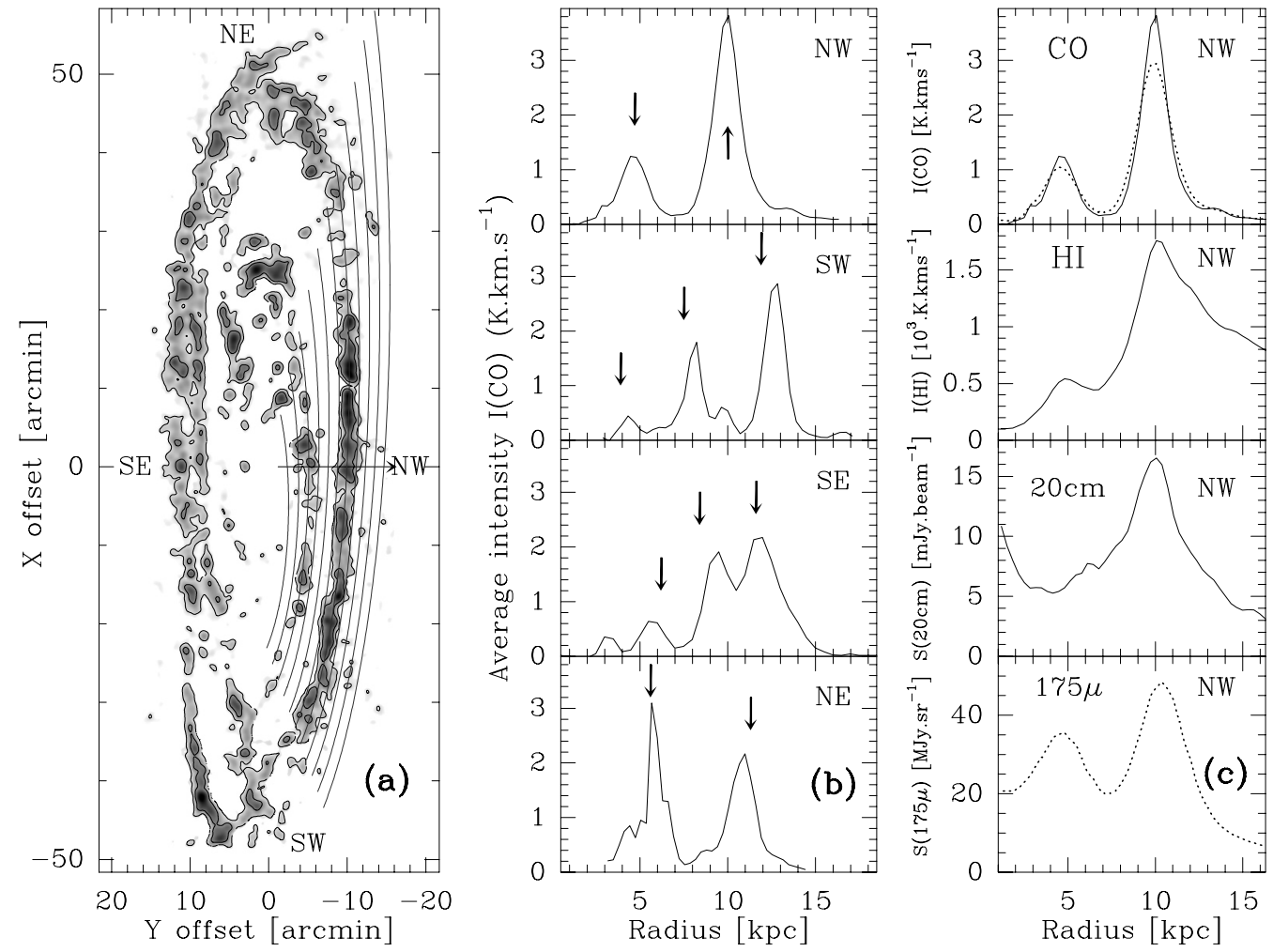

Fig. 4. a) A family of logarithmic spiral segments (defined in the plane of M31) fitting the CO arms in the NW quadrant, superimposed onto the velocity-integrated $\mathrm{CO}$ emission, $I_{1-0}$, smoothed to a resolution of $45^{\prime \prime}$. The spiral segments densely cover the NW quadrant between $Y=1^{\prime}$ and $Y=14^{\prime}$ on the minor axis (indicated by the arrow). b) Top: mean profile of the CO intensity across the $5 \mathrm{kpc}$ and $10 \mathrm{kpc}$ spiral arms, averaged along the spiral segments shown in Fig. 4a. Middle and Bottom: same as the upper figure for the SW, SE, and NE quadrants. The scale on the abscissa shows the galactocentric distance along the minor axis (where $1^{\prime}$ corresponds to $1050 \mathrm{pc}$ ) for the NW and SE quadrants, and along the major axis for the SW and NE quadrants (where 1' corresponds to $227 \mathrm{pc}$ ). The arrows indicate the points of intersection of the two-armed logarithmic spiral pattern with the minor or major axis; note that they do not always coincide with the CO arms. c) Comparison of the mean $\mathrm{CO}, \mathrm{HI}, 20 \mathrm{~cm}$ continuum, and $175 \mu \mathrm{m}$ continuum emission profiles in the NW quadrant. The full curves were obtained from maps at 45" resolution; the dotted curves in the top and bottom panels resulted from maps at $90^{\prime \prime}$ resolution. See text for further explanations. 
M31, the arm thickness perpendicular to the plane and the effect of beam-smoothing. Nieten (2001) attempted to disentangle these 3 effects for the NW part of the $10 \mathrm{kpc}$ arm and derived a "true" width of $500 \pm 100 \mathrm{pc}$ and an arm thickness of $150 \pm 50 \mathrm{pc}$.

We repeated the above analysis for the H I map of Brinks \& Shane (1984), smoothed to the same resolution of $45^{\prime \prime}$, for the $20 \mathrm{~cm}$ radio continuum map of Beck et al. (1998) at the original $45^{\prime \prime}$ resolution and for the $175 \mu \mathrm{m}$ map of Haas et al. 1998) at $90^{\prime \prime}$ resolution (see Fig. 4c). The arm-interarm contrast of the integrated $\mathrm{H}$ I-line brightness is $\simeq 4$, which is 5 times smaller than that of $\mathrm{CO}$ in the same disk section, and the apparent half-power arm width of $6^{\prime}$ is 3 times larger. In contrast to $\mathrm{CO}$ emission, $\mathrm{H}$ I emission is detected everywhere between the arms at $5 \mathrm{kpc}$ and $10 \mathrm{kpc}$. At $\lambda 20 \mathrm{~cm}$ the contrast is about 2.5 and the arm width is between the widths in $\mathrm{CO}$ and $\mathrm{HI}$. This was also noted by Berkhuijsen et al. (1993), who found that the width at $\lambda 20 \mathrm{~cm}$ corresponds to the width of the total gas arm, at a resolution of $75^{\prime \prime}$.

The molecular arms traced by $\mathrm{CO}$ are much narrower and thinner than the $\mathrm{H}$ I arms, and the arm-interarm contrast is much higher. This indicates that the molecular phase is short-lived compared to the lifetime of the H I gas.

From a comparison of $\mathrm{CO}$ emission and visual extinction in a wide strip centred on the SW bright arm, Neininger et al. (1998) concluded that $\mathrm{CO}(1-0)$ line emission is a good tracer of the molecular gas in M 31, including the interarm region. The $>5$ times higher arm-interarm ratio in $\mathrm{CO}$ compared to $\mathrm{H} \mathrm{I}$ thus implies that the molecular gas has almost vanished in the interarm regions. Yet molecular and atomic clouds have about the same velocity dispersion (see Sect. 5), have roughly the same response to the stellar potential and follow the same orbits with the same orbital velocity. This indicates that molecular clouds become mostly atomic when leaving the arms.

An accurate determination of the molecular cloud lifetimes would require a density-wave model of M 31 that explains the observed CO arm pattern. Such a model is outside the scope of the present paper, although a crude upper limit to this lifetime can be estimated from the $\mathrm{CO}$ and $\mathrm{H} \mathrm{I}$ arm-interarm ratios. Consider a gas cloud orbiting around M 31 's centre and crossing a spiral arm. From Fig. 4c we estimate that the total gas arminterarm ratio is $4-5$. This means that, due to streaming motions, the clouds stay 4-5 times longer inside the arms than they would if they followed purely circular orbits at a constant velocity. For an arm width of $0.5 \mathrm{kpc}$, a pitch angle of $8^{\circ}$, and an orbital velocity of $300 \mathrm{~km} \mathrm{~s}^{-1}$, the time spent in an arm is $5 \times 10^{7} \mathrm{yr}$, which is an upper limit to the age of the molecular clouds.

\subsection{Line ratios in selected regions}

Our observations of several selected regions on a finer grid yielded maps with high signal-to-noise ratio in the $(2-1)$ line as well as in (1-0). Two example maps of the velocity-integrated CO (2-1) line intensity (Fig. 5) cover some bright segments of spiral arms, about 2-4 kpc long: one located near the major axis about $6 \mathrm{kpc}$ from the centre (Fig. 5a), the other west of the main axis and about $9 \mathrm{kpc}$ from the centre (Fig. 5b). The ratio $R_{21}$ of the (2-1)-to-(1-0) line intensities is often regarded as a first indicator of excitation conditions in the clouds emitting the CO lines. To apply this test to our data, we smoothed the $(2-1)$ map to the same resolution $\left(23^{\prime \prime}\right)$ as the $(1-0)$ map, selected those points where both line intensities were above $3 \sigma$, and computed the line ratio. In the region of Fig. 5a we found an average ratio of $0.65 \pm 0.1$ with values reaching up to 1.0
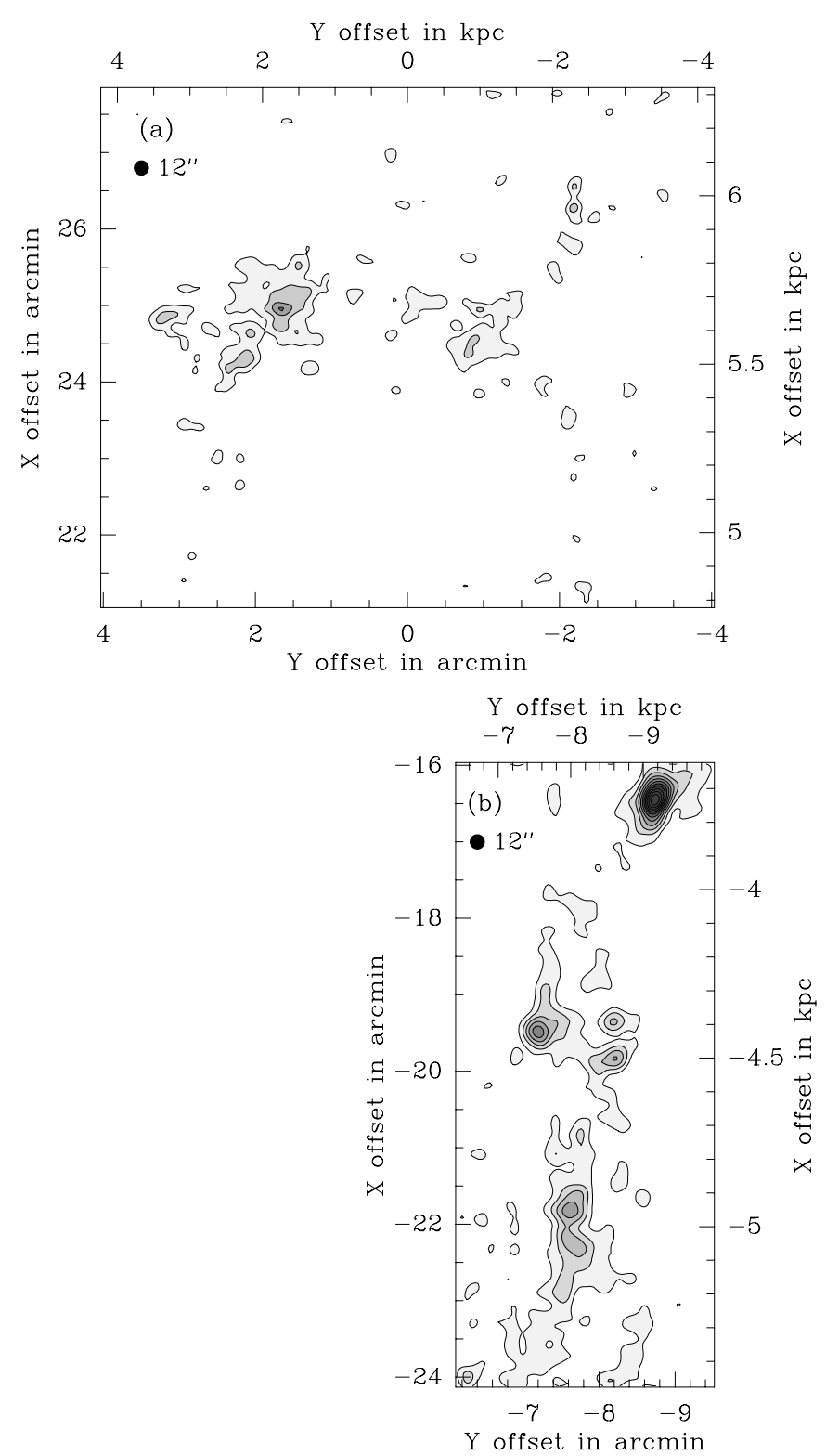

Fig. 5. Two maps showing the integrated emission of the ${ }^{12} \mathrm{CO}(2-1)$ transition at a resolution of $12^{\prime \prime}$. The contour lines correspond to 3,5 , $7, \ldots$ times the noise $\left(T_{\mathrm{mb}}\right.$ scale). a) Spiral arm on the major axis at a distance of $5.5 \mathrm{kpc}$ north of the centre; the noise is $0.7 \mathrm{~K} \mathrm{~km} \mathrm{~s}^{-1}$. b) Spiral arm region about $9 \mathrm{kpc}$ west of the centre; the noise is $1.2 \mathrm{~K} \mathrm{~km} \mathrm{~s}^{-1}$.

near D615 $(X, Y=25.5,3.0)^{3}$; in the clouds of Fig. $5 b$ we found an average of $0.5 \pm 0.1$ with ratios up to 0.7 around D84 $(X, Y=-16.5,-8.7)$.

These values are compatible with simple standard assumptions about the $\mathrm{CO}$ excitation: i.e., (i) both lines are optically thick; (ii) they have the same excitation temperature $T_{\mathrm{ex}}$ that equals the gas kinetic temperature $T_{\text {kin }}$; and (iii) they sample the same gas of uniform conditions. The expected line ratio under these conditions is $R_{21}=0.49$ for $T=T_{\mathrm{ex}}=T_{\text {kin }}=3.5 \mathrm{~K}, R_{21}=$ 0.67 for $T=6 \mathrm{~K}$, and $R_{21}=0.79$ for $T=10 \mathrm{~K}$. The line ratios we found in the spiral arms of M 31 are also similar to values found widely over the Milky Way (e.g., Sakamoto et al. 1997), as well as to those of 0.7 to 0.8 seen in M 51 (Garcia-Burillo et al. 1993) and 0.60 to 0.85 in NGC 891 (Garcia-Burillo et al. 1992). For these reasons the CO excitation in the bright regions

3 The dark cloud complexes (e.g. D615) are indicated with their appellation in Hodge's Atlas of the Andromeda Galaxy (Hodge 1981a). 
M31 CO HPBW=23" North (superposed on U band)

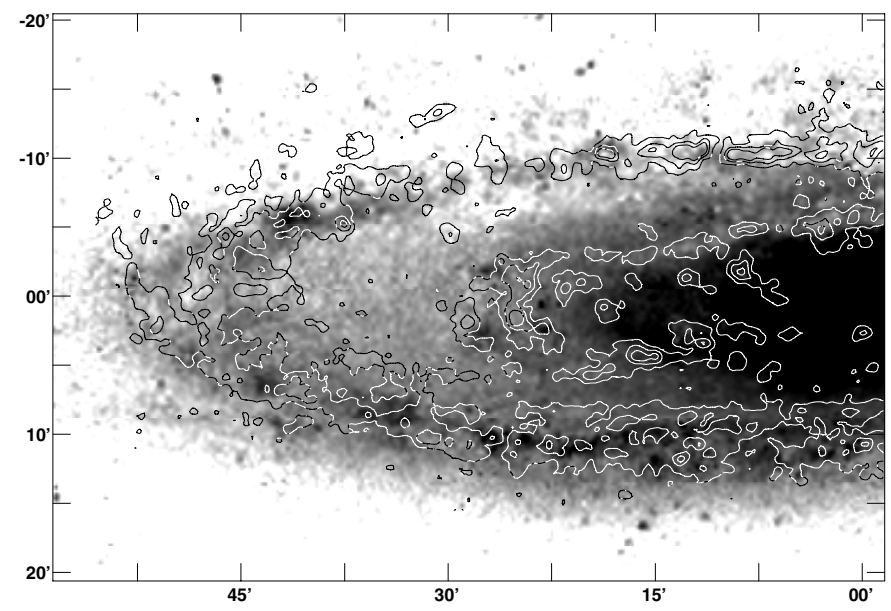

M31 CO HPBW=23" South (superposed on U band)

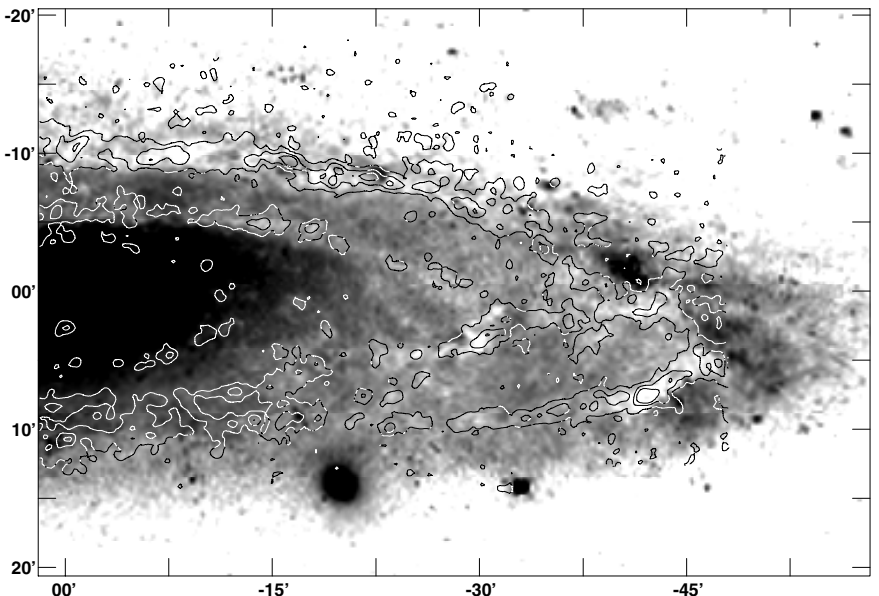

Fig. 6. Contours of ${ }^{12} \mathrm{CO}(1-0)$ emission from M 31 at $23^{\prime \prime}$ resolution on a U-plate of Walterbos \& Kennicutt (1988). The contour levels are 1 (=3× rms noise), 4 and $8 \mathrm{~K} \mathrm{~km} \mathrm{~s}^{-1}$. a) Northern half of M 31. b) Southern half of M 31 .

of our survey is clearly different from that of gas that shows very low "subthermal" values of $R_{21} \sim 0.2$ to $\sim 0.4$, as found, e.g., by Allen \& Lequeux (1993) and Loinard et al. (1995) towards some positions in M 31. Of course these papers mostly concern the positions of small clouds or weak emission, whereas our values are selected for positions with relatively strong lines and therefore probably high ratios $R_{21}$. We note, however, that Melchior et al. (2000) reported an $R_{21}$ ratio of 0.65 towards the weak CO complex associated with D395A, located only $350 \mathrm{pc}$ from the centre of M 31. This value is quite similar to the ratios that we find. A more complete study of $\mathrm{CO}$ excitation in M31 would have to include positions of weaker emission and also observations of optically thin CO isotopes, but this is beyond the scope of our present paper.

\section{Comparison of $\mathrm{CO}$ with other gas and dust tracers}

The high resolution data presented in this paper allow detailed comparisons with other tracers of the gas and the dust. Figure 6 shows contours of $\mathrm{CO}$ brightness superimposed onto a $U$-band image (Walterbos \& Kennicutt 1988). The CO traces the dust lanes closely, especially in the western part of M $31\left(Y<0^{\prime}\right)$ where the dust lanes stand out against the light of the stellar bulge. The extended dark region around $X=30^{\prime}, Y=-7^{\prime}$ shows little $\mathrm{CO}$, but this area has low brightness in many constituents of M 31 (see Fig. 8). The weak inner CO arm crossing the major axis near $X=-12^{\prime}$ appears to coincide with a narrow dust lane seen at $7.7 \mu \mathrm{m}$ by Willaime et al. (2001), who observed the central part of M 31 with ISOCAM LW6 (see Fig. 7). The CO clouds near the major axis between $X=0^{\prime}$ and $10^{\prime}$ also coincide with dust features at $7.7 \mu \mathrm{m}$, but not all dust features are seen in CO.

Figure 8 collects the observed brightness distributions of $\mathrm{CO}$, $\mathrm{HI}$, total neutral gas, FIR at $175 \mu \mathrm{m}$ and $20 \mathrm{~cm}$ radio continuum. The $175 \mu \mathrm{m}$ emission mainly traces cold dust at temperatures near $16 \mathrm{~K}$ (Haas et al. 1998). The difference between the distributions of $\mathrm{CO}$ and $\mathrm{HI}$ is striking. The $\mathrm{HI}$ arms are much smoother and wider than the $\mathrm{CO}$ arms and weak H I emission is seen nearly everywhere outside the spiral arms. We obtained the distribution of the total gas column density, $N_{\text {gas }}=N(\mathrm{HI})+$ $2 N\left(\mathrm{H}_{2}\right)$, after smoothing the $\mathrm{CO}$ map to the angular resolution of $24^{\prime \prime} \times 36^{\prime \prime}$ of the H I map (Brinks \& Shane 1984). We used the

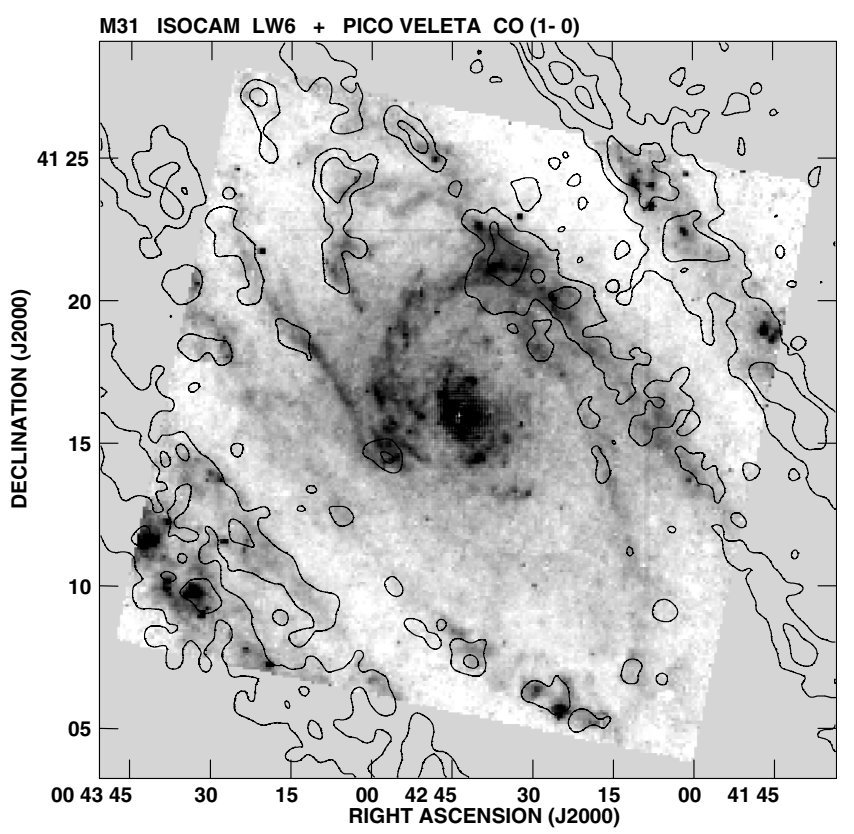

Fig. 7. Contours of ${ }^{12} \mathrm{CO}(1-0)$ emission from $\mathrm{M} 31$ at $23^{\prime \prime}$ resolution on the dust emission at $\lambda 7.7 \mu \mathrm{m}$ observed in the central bulge (Willaime et al. 2001, Fig. 2). The contour levels are $1(=3 \times$ rms noise), 4 and $10 \mathrm{~K} \mathrm{~km} \mathrm{~s}^{-1}$. The white dot near the centre of the image indicates the centre of M31. Note the alignment of CO clouds along the faint elliptical ring south of the nucleus.

Galactic conversion factor $X_{\mathrm{CO}}=N\left(\mathrm{H}_{2}\right) / \int T_{\mathrm{mb}}\left({ }^{12} \mathrm{CO}_{1-0}\right) \mathrm{d} v=$ $1.9 \times 10^{20} \mathrm{~mol} \mathrm{~cm}^{-2}\left(\mathrm{~K} \mathrm{~km} \mathrm{~s}^{-1}\right)^{-1}$ given by Strong \& Mattox (1996), which was assumed to be constant across the CO map. This conversion factor is supported by $1.2 \mathrm{~mm}$ observations of the thermal emission from dust in two extended regions in the disk (Zylka \& Guélin, in prep.) and by virial mass estimates of several molecular cloud complexes in M31 (Muller 2003; Guélin et al. 2004). The H I column density was calculated from the relation $N(\mathrm{H} \mathrm{I}) / \int T_{\mathrm{b}} \mathrm{d} v=1.82 \times 10^{18}$ at cm $^{-2}$, valid for optically thin lines. This relation may lead to a significant underestimate of $N(\mathrm{HI})$ if $T_{\mathrm{b}}>70 \mathrm{~K}$ (Braun \& Walterbos 1992), i.e. at the crest of the arms. The distribution of the total gas calculated this way is shown in Fig. 8c.

Comparing the various distributions in Fig. 8, we notice that the nuclear area is only prominent at $175 \mu \mathrm{m}$ and $\lambda 20 \mathrm{~cm}$ radio 


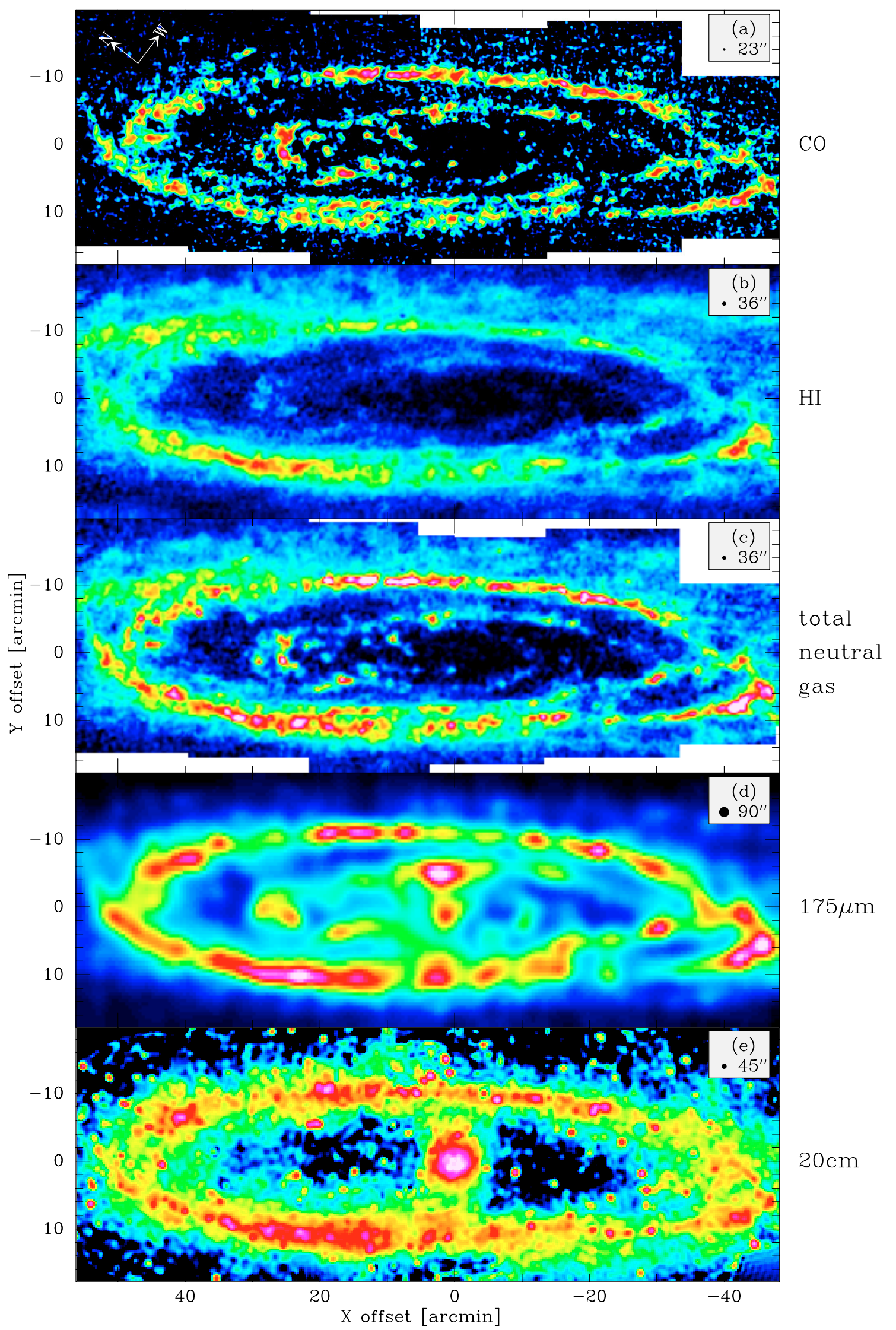

Fig. 8. Distributions of neutral gas, cold dust and radio continuum in $\mathrm{M} 31$. From top to bottom: a) emission observed in the ${ }^{12} \mathrm{CO}(1-0)$ line (this paper); b) emission observed in the $\mathrm{HI}$ line (Brinks \& Shane 1984); c) emission from the total neutral gas, $N(\mathrm{HI})+2 N\left(\mathrm{H}_{2}\right)$, with $X_{\mathrm{CO}}=$ $1.9 \times 10^{20} \mathrm{~mol} \mathrm{~cm}^{-2}\left(\mathrm{~K} \mathrm{~km} \mathrm{~s}^{-1}\right)^{-1}$ (see text); d) emission from cold dust at $\lambda 175 \mu \mathrm{m}$ (Haas et al. 1998); e) radio continuum emission at $\lambda 20 \mathrm{~cm}$ (Beck et al. 1998). The half-power beamwidth is indicated in the upper right-hand corner of each map. The intensities at the maximum near $X, Y=-42^{\prime}, 8^{\prime}$ (white-red) and the minimum near $X, Y=-30^{\prime}, 10^{\prime}$ (blue-green) in each map are: a) 6.7 and 0.8 in $10^{21}$ at $\mathrm{cm}^{-2}$, b) 6.5 and 2.0 in $10^{21} \mathrm{at} \mathrm{cm}^{-2}$, c) 12.1 and 2.6 in $10^{21}$ at cm${ }^{-2}$, d) 85 and 27 in MJy/sr, e) 2.9 and 0.7 in Jy/beam area. The M 31 coordinates are based on the centre position $(\alpha, \delta)_{50}=\left(0^{\mathrm{h}} 40^{\mathrm{m}} 00^{\varsigma} 3,41^{\circ} 00^{\prime} 03^{\prime \prime}\right)$ and a position angle of the major axis $\mathrm{PA}=37.7$ (see footnote 1$)$. 


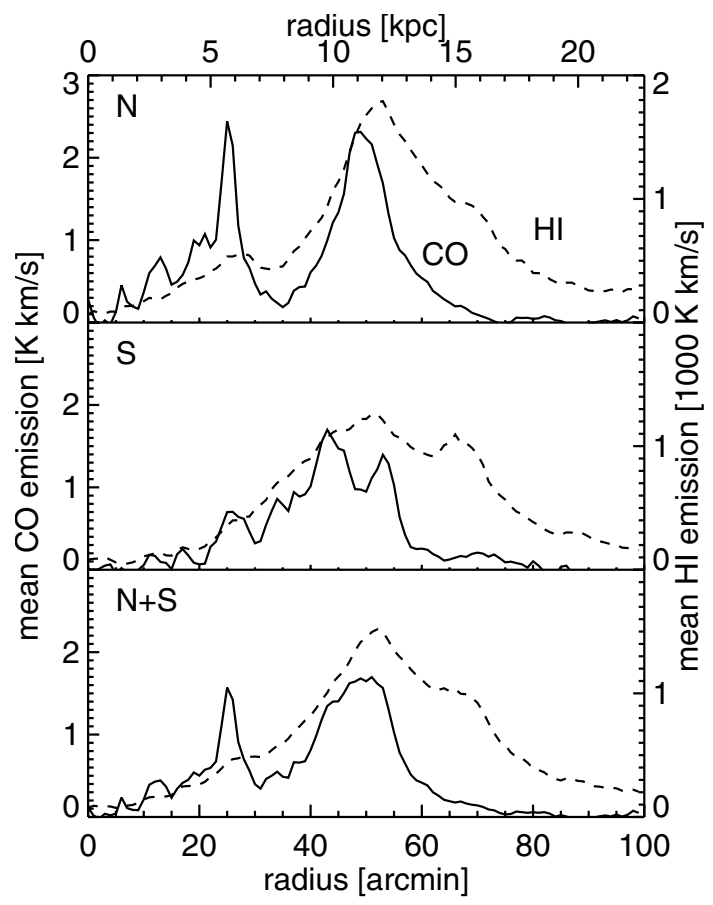

Fig. 9. Radial profiles of $I_{1-0}$ (full lines, left-hand scale) and $I_{\mathrm{H}}$ (dashed lines, right-hand scale) for the northern half $(\mathrm{N})$, the southern half $(\mathrm{S})$ and the full area of M31 $(\mathrm{N}+\mathrm{S})$. The profiles show intensities along the line of sight averaged in circular rings of $1^{\prime}$ width in the plane of M 31; typical standard deviations are $0.01 \mathrm{~K} \mathrm{~km} \mathrm{~s}^{-1}$ and $5 \mathrm{~K} \mathrm{~km} \mathrm{~s}^{-1}$ for the $\mathrm{CO}$ and the $\mathrm{HI}$ profiles, respectively. The $\mathrm{CO}$ profiles were obtained from the map at $23^{\prime \prime}$ resolution (Fig. 1) and the H I profiles from the map of B\&S at $24^{\prime \prime} \times 36^{\prime \prime}$ resolution. The limited extent of the CO map on the major axis, $R=55^{\prime}$ in the north and $R=50^{\prime}$ in the south, reduces the intensities of the profiles of $I_{1-0}$ beyond these radii by a few percent.

continuum, but all distributions in Fig. 8 show the pronounced ring of emission at about $10 \mathrm{kpc}$ from the centre, where most of the star-formation regions are also located. The spiral arm closer to the nucleus, clearly visible in $\mathrm{CO}$ and at $175 \mu \mathrm{m}$, is hardly seen in $\mathrm{HI}$ and radio continuum. This indicates that the CO-to$\mathrm{H}$ I brightness ratio increases towards smaller radii. Only the $\mathrm{HI}$ and the $175 \mu \mathrm{m}$ distributions show extended weak emission between the spiral arms. Thus the $\mathrm{CO}$ emission is concentrated in regions of the denser clouds seen at $175 \mu \mathrm{m}$ and in $\mathrm{HI}$, located in the spiral arms, and does not trace weak and extended interarm emission at $175 \mu \mathrm{m}$, which is especially visible at $Y>0^{\prime}$. In Sect. 4.2 we present the first results of a correlation study between $\mathrm{CO}, \mathrm{HI}$ and $175 \mu \mathrm{m}$. Radial distributions and the gasto-dust ratios are shown in Sect. 4.1.

The radio continuum emission at $\lambda 20 \mathrm{~cm}$ (Beck et al. 1998) is also concentrated in the main spiral arms that form the emission ring. Since at this wavelength most of the emission is nonthermal, magnetic fields and cosmic rays are concentrated in this ring. Using the CO data of Koper et al. (1991), Berkhuijsen (1997) compared nonthermal emission with CO and H I data at a resolution of $9^{\prime}$. Although significant correlations indeed exist at this resolution, detailed correlations with the new data are required to enable an interpretation in terms of the coupling between magnetic fields and gas. This will be the subject of a forthcoming study.

\subsection{Radial distributions}

In Fig. 9 we show the radial variations of the observed $\mathrm{CO}$ and $\mathrm{H}$ I brightness distributions averaged in 230 pc-wide circular rings in the plane of $\mathrm{M} 31$ (i.e. 1' on the major axis). Because

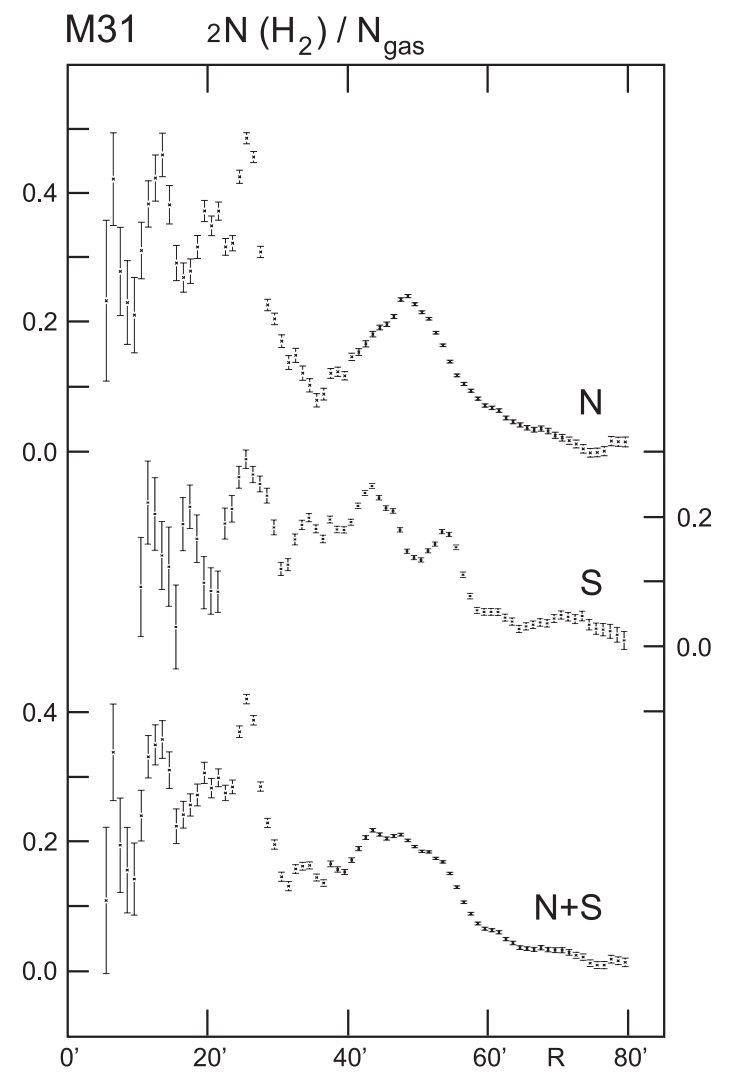

Fig. 10. Radial variation of the molecular gas fraction $2 N\left(\mathrm{H}_{2}\right) / N_{\text {gas }}$ for the northern half ( $\mathrm{N}$, upper left-hand scale), the southern half ( $\mathrm{S}$, right-hand scale) and the full area of $\mathrm{M} 31(\mathrm{~N}+\mathrm{S}$, lower lefthand scale), where $N_{\text {gas }}=N(\mathrm{HI})+2 N\left(\mathrm{H}_{2}\right)$ and $X_{\mathrm{CO}}=1.9 \times$ $10^{20} \mathrm{~mol} \mathrm{~cm}^{-2}\left(\mathrm{~K} \mathrm{~km} \mathrm{~s}^{-1}\right)^{-1}$ (Strong \& Mattox 1996). The CO map was smoothed to the resolution of $24^{\prime \prime} \times 36^{\prime \prime}$ of the H I map before the molecular fractions were computed from the column densities averaged in circular rings of $1^{\prime}$ width in the plane of M31.

the spiral arms in M31 are tightly wound, the narrow CO arms clearly stand out in the radial distributions. In the northern part $\left(X>0^{\prime}\right)$, the average intensity of the inner arm at $R \simeq 25^{\prime}$ is comparable to that of the arms forming the bright emission ring at $R \simeq 50^{\prime}$, whereas this inner arm is much weaker in the southern part $\left(X<0^{\prime}\right)$. The radial profiles of $\mathrm{H}$ I are much wider than those of $\mathrm{CO}$, but the inner arm and main ring are still indicated. The H I disk extends as far as $R \simeq 150^{\prime}, 34 \mathrm{kpc}$ from the nucleus (Emerson 1974), about 2 times further than the molecular gas in the map of Koper et al. (1991).

Our CO profiles of the southern half show only half as much emission as those in Fig. 10d of Loinard et al. (1999), whereas the H I profiles are nearly identical. This may indicate a difference in the adopted $\mathrm{CO}$ intensity scales, e.g. in the calibration or in the corrections for beam efficiencies. In Sect. 4.3 we find close agreement of our CO intensity scale with that of the $1.2-\mathrm{m}$ telescope at the Center for Astrophysics (Dame et al. 1993), to which the standard Galactic $X_{\mathrm{CO}}$ is calibrated. We also find that even after smoothing our map to the effective resolution of $1^{\prime}$ of the map of Loinard et al., the half-intensity width of the $\mathrm{CO}$ arms in their Figs. 5 and $6 a$ is about 1.5 times larger than in our map. We may attribute this discrepancy to the much higher error beam of the FCRAO telescope compared to that of the IRAM 30-m dish.

The fraction of molecular-to-total gas, $2 N\left(\mathrm{H}_{2}\right) / N_{\text {gas }}$, generally decreases with increasing distance from the centre (see Figs. 10 and 11). The highest values occur on the weak inner 


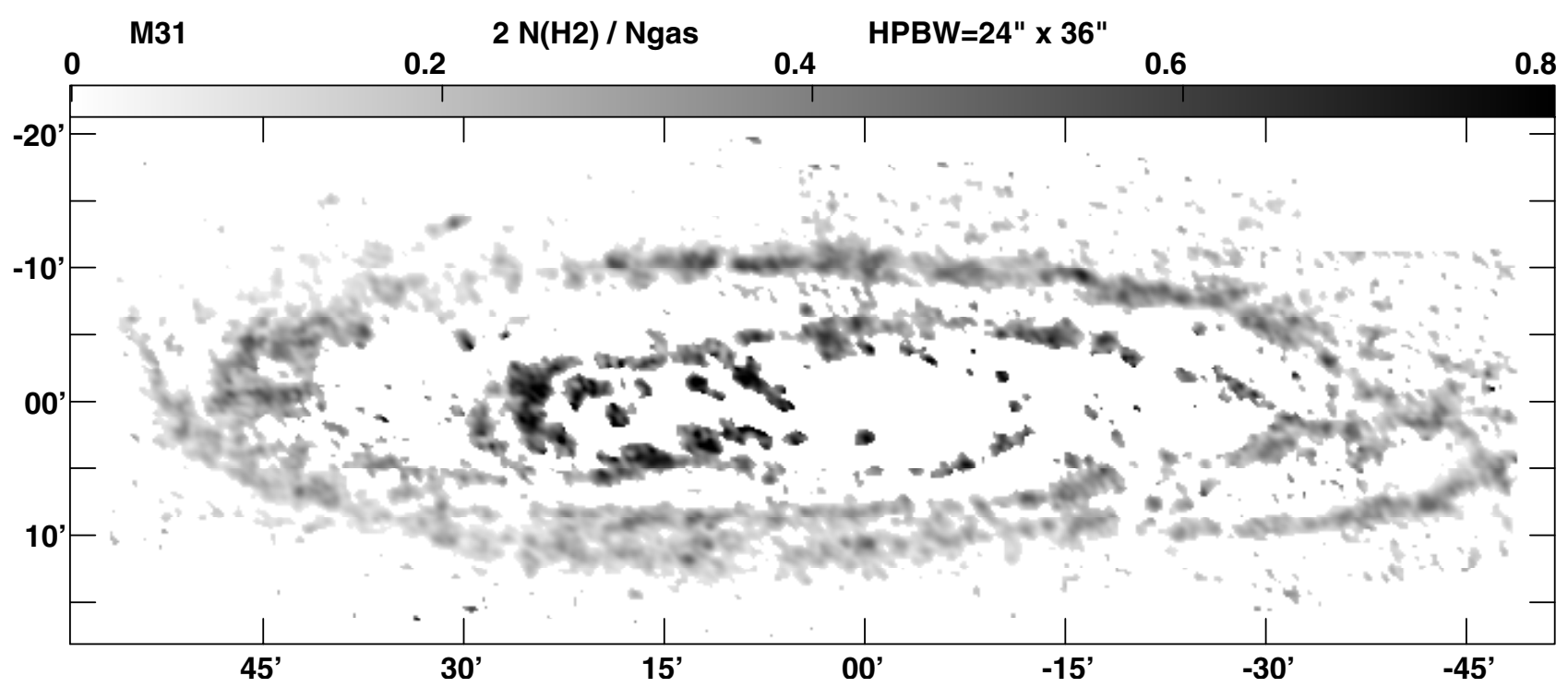

Fig. 11. Distribution of the molecular gas fraction $2 N\left(\mathrm{H}_{2}\right) / N_{\text {gas }}$ in $\mathrm{M} 31$ at a resolution of $24^{\prime \prime} \times 36^{\prime \prime}$. Only data points larger than $3 \times$ rms noise in the $\mathrm{CO}$ and $\mathrm{H}$ I maps were used. We assumed $N_{\text {gas }}=N(\mathrm{H} \mathrm{I})+2 N\left(\mathrm{H}_{2}\right)$ and a constant value of $X_{\mathrm{CO}}=1.9 \times 10^{20} \mathrm{~mol} \mathrm{~cm}^{-2}\left(\mathrm{~K} \mathrm{~km} \mathrm{~s}^{-1}\right)^{-1}(\mathrm{Strong} \mathrm{\&}$ Mattox 1996).

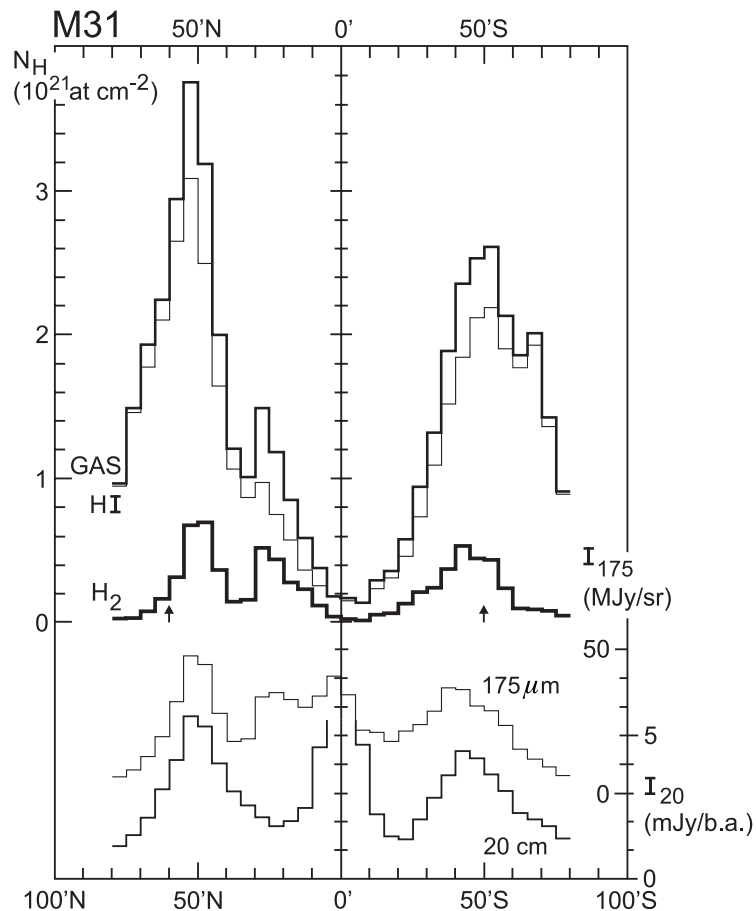

Fig. 12. Radial variations for the northern (left panel) and the southern (right panel) halves of $\mathrm{M} 31$ of $N_{\text {gas }}, N(\mathrm{HI})$ and $2 N\left(\mathrm{H}_{2}\right)$ (left-hand scale), as well as of $I_{175 \mu \mathrm{m}}$ (inner right-hand scale) and $I(20 \mathrm{~cm})$ (outer right-hand scale) obtained from the distributions in Fig. 8. The gas maps and the radio continuum map were smoothed to the resolution of $90^{\prime \prime}$ of the $175 \mu \mathrm{m}$ map. The line-of-sight surface brightnesses were averaged in circular rings around the centre of $5^{\prime}$ width in the plane of M31. The two arrows indicate the extent of the CO map on the major axis.

arm at $R \simeq 12^{\prime}(2-3 \mathrm{kpc})$ and on the bright inner arm at $R \simeq 25^{\prime}$ $(5-6 \mathrm{kpc})$ where the molecular fraction is nearly 0.5 in the north and about 0.25 in the south (see Fig. 10). On the bright ring at $R \simeq 50^{\prime}$, the molecular fraction is about 0.2 both in the northern and in the southern parts, although the northern part of the ring contains about 1.5 times more neutral gas than the southern one.

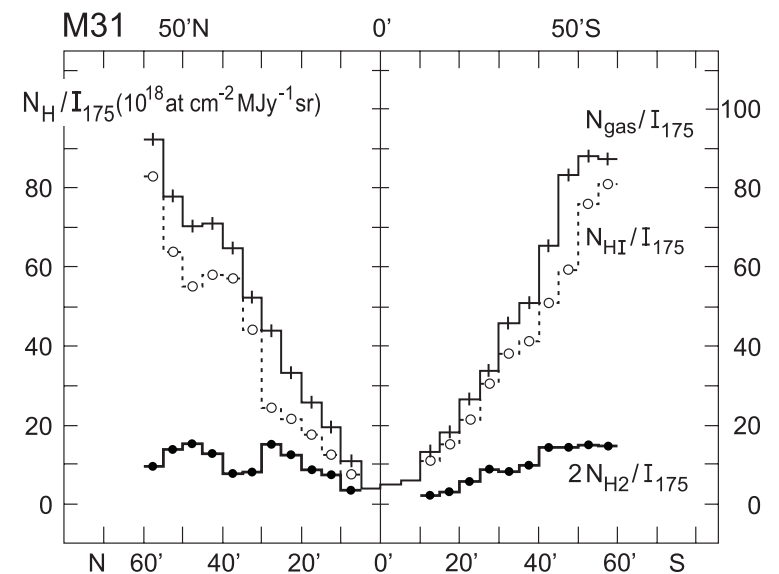

Fig. 13. Radial variation of apparent gas-to-dust ratios for the northern (left panel) and the southern halves (right panel) of M31 derived from the profiles in Fig. 12. Thin full line $-N_{\text {gas }} / I_{175}$, dashed line $N(\mathrm{H} \mathrm{I}) / I_{175}$ and thick full line $-2 N\left(\mathrm{H}_{2}\right) / I_{175}$. As before, $N_{\text {gas }}=N(\mathrm{HI})+$ $2 N\left(\mathrm{H}_{2}\right)$ and a constant value of $X_{\mathrm{CO}}=1.9 \times 10^{20} \mathrm{~mol} \mathrm{~cm} \mathrm{~cm}^{-2}\left(\mathrm{~K} \mathrm{~km} \mathrm{~s}^{-1}\right)^{-1}$ (Strong \& Mattox 1996) were used. Note the increase in $2 N\left(\mathrm{H}_{2}\right) / I_{175}$ on the molecular arm at $R \simeq 25^{\prime}$ and on the main emission ring at $R \simeq 50^{\prime}$.

The averages in circular rings underestimate the ratios on the arms. Figures $4 \mathrm{c}$ and 11 yield a decrease from $\sim 0.6$ on the arms at $5 \mathrm{kpc}$ to $0.3-0.4 \mathrm{on}$ the arms at $10 \mathrm{kpc}$. The observed values of $2 N\left(\mathrm{H}_{2}\right) / N_{\text {gas }}$ and its radial decrease are typical of nearby galaxies (Honma et al. 1995). The decrease in the molecular fraction in Fig. 10 confirms the decrease in the fraction of molecular gas mass along the major axis of M 31 reported by Dame et al. (1993). Along the arms the molecular fraction varies considerably (see Fig. 11). The highest fraction detected is 0.96 for a cloud near the northern major axis at $X=13^{\prime}, Y=-1^{\prime}$, which seems purely molecular.

We smoothed the distributions of $\mathrm{CO}, \mathrm{HI}$, total gas and $20 \mathrm{~cm}$ radio continuum in Fig. 8 to the angular resolution of 90" of the $175 \mu \mathrm{m}$ map and compare their radial distributions in Fig. 12. In all constituents the main ring at $R \simeq 50^{\prime}$ is brighter in the north than in the south. The pronounced molecular arm in 
M31 1.0 $\log ($ I175 / Ngas )
1.5
HPBW=90"

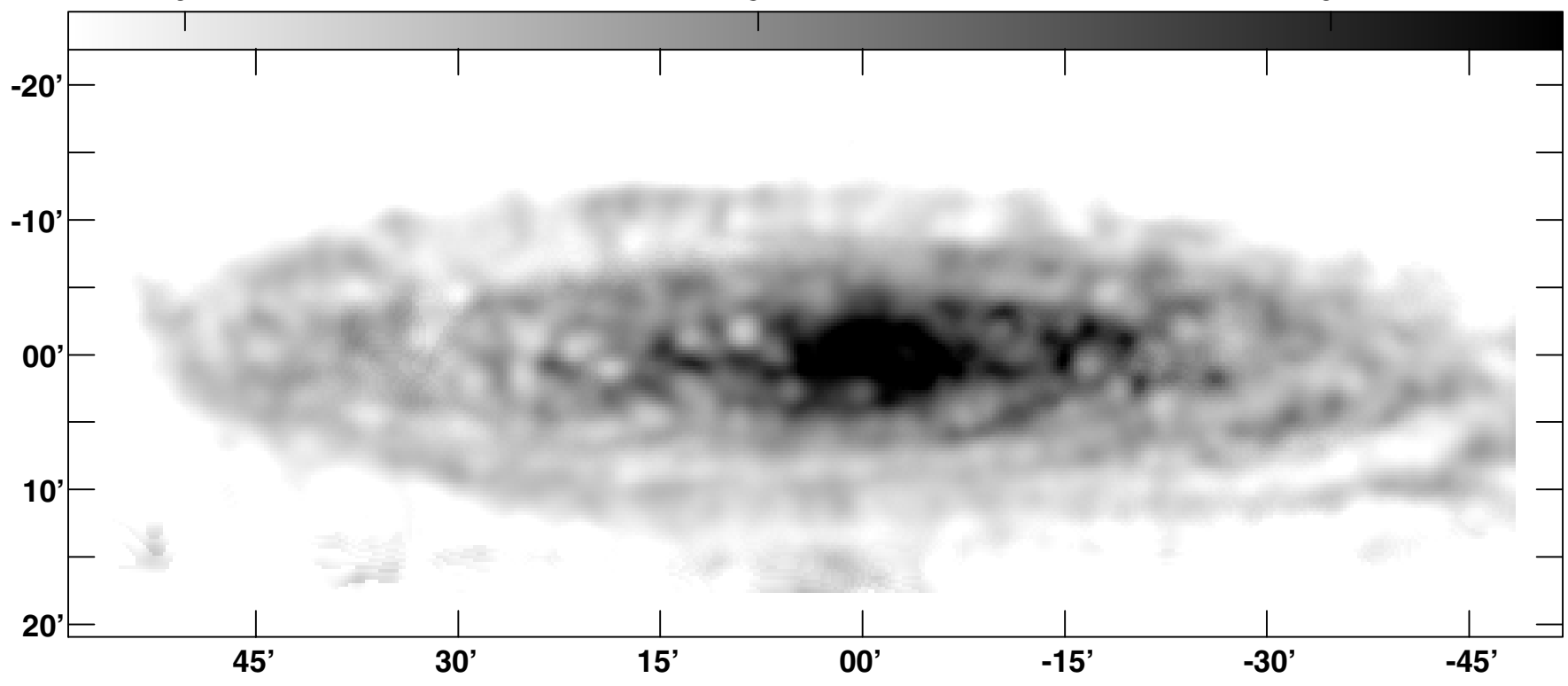

Fig. 14. Distribution of the apparent dust-to-total gas ratio, $I_{175} / N_{\text {gas }}\left(\mathrm{MJy} \mathrm{sr}^{-1}\right) /\left(10^{21} \mathrm{at} \mathrm{cm}^{-2}\right)$, in $\mathrm{M} 31$ at $90^{\prime \prime}$ resolution. Local minima (holes) are caused by enhancements in $N\left(\mathrm{H}_{2}\right)$ (compare to Figs. 1a or 11).

the north at $R \simeq 25^{\prime}$ is invisible in radio continuum, possibly because of a lack of relativistic electrons (Moss et al. 1998). When we disregard the central region $R<10^{\prime}$, the profiles of molecular gas and $175 \mu \mathrm{m}$ emission from cold dust are the most alike.

Using the profiles in Fig. 12, we calculated the apparent gasto-dust ratios $2 N\left(\mathrm{H}_{2}\right) / I_{175}, N(\mathrm{H} \mathrm{I}) / I_{175}$ and $N_{\text {gas }} / I_{175}$ as a function of radius presented in Fig. 13. The ratio $2 N\left(\mathrm{H}_{2}\right) / I_{175}$ is clearly enhanced in the spiral arms at $R \simeq 25^{\prime}$ and $R \simeq 50^{\prime}$, especially in the north, whereas $N(\mathrm{H}$ I $) / I_{175}$ continuously increases steeply from the centre outwards by nearly a factor 20 . As H I is the dominant gas component, the apparent total gas-to-dust ratio $N_{\text {gas }} / I_{175}$ increases by about a factor 20 between $R=0^{\prime}$ and $R=60^{\prime}$. However, the physical reality of this strong increase may be questionable. First, the $\mathrm{H}$ I line opacity changes with radius; second, the $X_{\mathrm{CO}}$ conversion factor may vary with radius; third, $I_{175}$ may not reflect the dust column density if the dust absorption cross section and, especially, the dust temperature vary with radius.

The first two causes are unlikely. Braun \& Walterbos (1992) showed that the atomic gas temperature in M 31 increases towards larger radii, while the $21-\mathrm{cm}$ line opacity decreases. This variation, however, is less than $\sim 20 \%$ and cannot account for the strong gradient in $N(\mathrm{HI}) / I_{175}$.

The behaviour of $X_{\mathrm{CO}}$ with radius has been the subject of several studies of the Milky Way and nearby galaxies (Wilson 1995; Sodroski et al. 1995; Strong et al. 2004). The value of $X_{\mathrm{CO}}$ is found to increase with increasing radius, perhaps in relation to decreasing metallicity. Such an increase, if present at all in M 31, would only enhance the radial variations in $N\left(\mathrm{H}_{2}\right) / I_{175}$ and $N_{\text {gas }} / I_{175}$.

A study of 50 bright cloud complexes at a resolution of at least $12^{\prime \prime}$, which is high enough to alleviate rotation velocity gradients, at radial distances between 5 and $12 \mathrm{kpc}$ (Muller 2003; Guélin et al. 2004) yield $X_{\mathrm{CO}}=(1-5) \times$ $10^{20} \mathrm{~mol} \mathrm{~cm}^{-2}\left(\mathrm{~K} \mathrm{~km} \mathrm{~s}^{-1}\right)^{-1}$ without any radial dependence. Melchior et al. (2000) and Israel et al. (1998) find similar values for two complexes at $R=0.35 \mathrm{kpc}$ and $R=2.5 \mathrm{kpc}$. Hence, except perhaps for the observation by Allen \& Lequeux (1993) of a dark cloud (D268, $R=2.5 \mathrm{kpc}$ ) with low CO luminosity and large linewidth, which may not be in equilibrium, there is no evidence of a radial variation of $X_{\mathrm{CO}}$ in M 31 .

It is more difficult to rule out a variation of the dust temperature $T_{\mathrm{d}}$ with radius. The thermal emission of cold dust at $175 \mu \mathrm{m}$ varies like $T_{\mathrm{d}}$ to the power $4-5$, so even a mild decrease in $T_{\mathrm{d}}$ outwards could cause a strong decrease in $I_{175}$. In fact, the apparent increase in $N_{\text {gas }} / I_{175}$ from 1 to $14 \mathrm{kpc}$ could be explained by a decrease in the dust temperature from $\sim 25 \mathrm{~K}$ to $15 \mathrm{~K}$. Only extensive mapping of this emission at longer wavelengths will allow us to discriminate between a temperature effect and a dustto-gas variation.

An increase in the apparent gas-to-dust ratio with increasing radius in M 31 was found by several authors from comparisons of $N(\mathrm{HI})$ and optical or UV extinction (Bajaja \& Gergely 1977; Walterbos \& Kennicutt 1988; Xu \& Helou 1996; Nedialkov et al. 2000; Savcheva \& Tassev 2002). From a study of globular clusters, Savcheva \& Tassev found a similar increase in the apparent gas-to-dust radio as we do. The distribution across M 31 of the reversed ratio, i.e. the apparent dust-to-total gas ratio $I_{175} / N_{\text {gas }}$, is shown in Fig. 14. The general decrease from the centre outwards is clearly visible. The ratio $I_{175} / N_{\text {gas }}$ tends to be low on the gas arms where the $\mathrm{CO}$ emission is strong, and even the many roundish minima in the distribution of $I_{175} / N_{\text {gas }}$ coincide with CO clouds.

It is interesting to note that on the brightest spot of emission at $175 \mu \mathrm{m}$, about $4^{\prime}$ west of the nucleus, the value of $I_{175} / N_{\text {gas }}$ is normal for that radius. This bright emission comes from one of the coldest dust clouds in M 31 analysed by Schmidtobreick et al. (2000). Willaime et al. (2001) showed that this cloud is located at a junction or superposition of several thin dust lanes seen at $7.7 \mu \mathrm{m}$ (see Fig. 7), which may explain its normal dustto-gas ratio. 

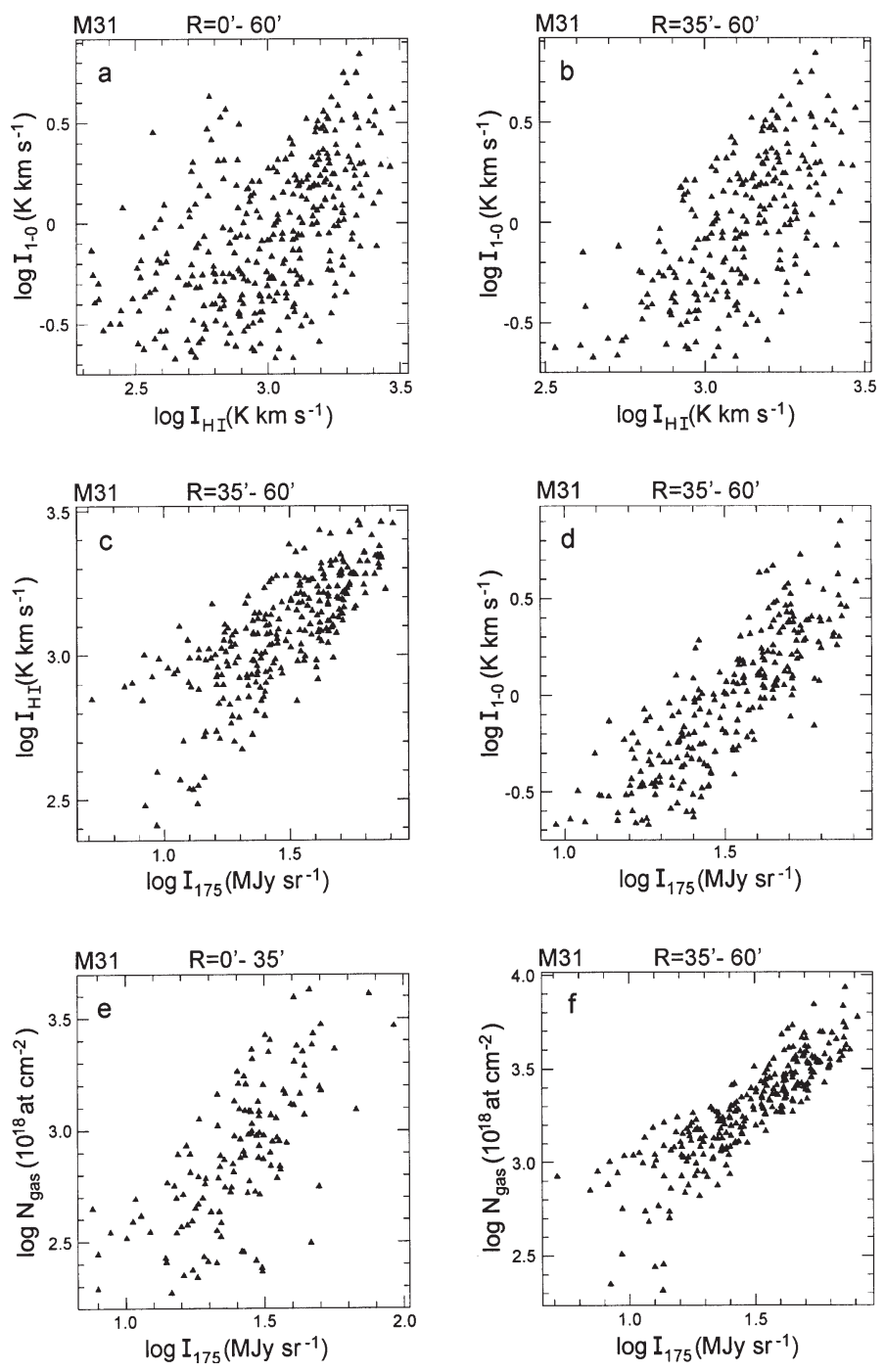

Fig. 15. Examples of correlations between neutral gas and cold dust $(16 \mathrm{~K})$ at $90^{\prime \prime}$ resolution for two radial ranges in M 31 . Only independent points (1.67 $\times$ HPBW apart) above $3 \times$ rms noise are used, in panels a), b), d) determined by the noise in the $\mathrm{CO}$ map and in c), e), f) by that in the H I map. The resulting fits of the bisector are given in Table 2 . a) $I_{1-0}$ as a function of $I_{\mathrm{H}}$ for $R=0^{\prime}-60^{\prime}$, and b) for $R=35^{\prime}-60^{\prime}$. The secondary maximum in a) at $\log I_{\mathrm{HI}} \simeq 2.8$ is due to the inner arm at $R \simeq 25^{\prime}$. c) $I_{\mathrm{HI}}$ as a function of $I_{175}$ for $R=35^{\prime}-60^{\prime}$. The two branches visible at $\log I_{175}<1.3$ result from the strong radial gradient in $N\left(\mathrm{HI}\right.$ ) $/ I_{175}$ (see Fig. 12). d) $I_{1-0}$ as a function of $I_{175}$ for $R=35^{\prime}-60^{\prime}$. e) $N_{\text {gas }}$ as a function of $I_{175}$ for $R=0^{\prime}-35^{\prime}$ and f) for $R=35^{\prime}-60^{\prime}$. Note that the extent of the logarithmic scales varies between panels.

\subsection{Correlations between the distributions of $\mathrm{CO}, \mathrm{HI}$ and FIR}

In Sect. 3.2 we compared the $\mathrm{CO}$ and $\mathrm{HI}$ distributions across the spiral arms. We now compare the general distribution of $\mathrm{CO}$ with that of $\mathrm{HI}$ and each of them with the $175 \mu \mathrm{m}$ FIR emission using the $\mathrm{CO}$ and $\mathrm{HI}$ maps smoothed to $90^{\prime \prime}$, the resolution of the FIR map. At this resolution the $\mathrm{CO}$ and $\mathrm{HI}$ arms near $R=10 \mathrm{kpc}$ merge to a broad emission ring, similar to the one seen at $175 \mu \mathrm{m}$. The comparison was restricted to radii $R<60^{\prime}$ $(=13.6 \mathrm{kpc})$ and intensities above $3 \times$ the rms noise at $90^{\prime \prime}$ resolution. Therefore, the noise in the $\mathrm{CO}$ map determined the
Table 2. Correlations between CO, H I and $175 \mu \mathrm{m}$.

\begin{tabular}{llrcccr}
\hline \hline$X$ & $Y$ & \multicolumn{1}{c}{$\Delta R$} & \multicolumn{2}{c}{$\log Y=a \log X+b^{1)}$} & \multicolumn{1}{c}{ Correl. } & $N^{1)}$ \\
& & \multicolumn{1}{c}{$\left({ }^{\prime}\right)$} & \multicolumn{1}{c}{$\mathrm{a}$} & \multicolumn{1}{c}{$\mathrm{b}$} & coeff. $\rho$ & \\
\hline$I_{\mathrm{HI}}{ }^{a}$ & $I_{1-0}{ }^{a}$ & $0-35$ & $2 \pm 5$ & $-5 \pm 20$ & $0.3 \pm 0.1$ & 90 \\
& & $35-60$ & $1.7 \pm 1.0$ & $-5.2 \pm 2.4$ & $0.61 \pm 0.04$ & 234 \\
& & $0-60$ & $1.3 \pm 0.5$ & $-4.1 \pm 1.4$ & $0.50 \pm 0.04$ & 324 \\
$I_{175}{ }^{b}$ & $I_{1-0}{ }^{a}$ & $0-35$ & $1.6 \pm 1.1$ & $-2.53 \pm 0.63$ & $0.56 \pm 0.07$ & 94 \\
& & $35-60$ & $1.57 \pm 0.20$ & $-2.39 \pm 0.16$ & $0.81 \pm 0.02$ & 234 \\
& & $0-60$ & $1.60 \pm 0.22$ & $-2.47 \pm 0.16$ & $0.75 \pm 0.02$ & 328 \\
$I_{175}{ }^{b}$ & $I_{\mathrm{HI}}{ }^{a}$ & $0-35$ & $0.8 \pm 1.8$ & $1.4 \pm 2.2$ & $0.51 \pm 0.07$ & 125 \\
& & $35-60$ & $0.84 \pm 0.27$ & $1.85 \pm 0.44$ & $0.75 \pm 0.03$ & 264 \\
& & $0-60$ & $1.09 \pm 0.32$ & $1.36 \pm 0.42$ & $0.54 \pm 0.04$ & 388 \\
$I_{175}{ }^{b}$ & $N_{\text {gas }}{ }^{c}$ & $0-35$ & $1.42 \pm 0.53$ & $0.89 \pm 0.60$ & $0.68 \pm 0.05$ & 134 \\
& & $35-60$ & $1.12 \pm 0.14$ & $1.64 \pm 0.22$ & $0.85 \pm 0.02$ & 263 \\
& & $0-60$ & $1.23 \pm 0.19$ & $1.27 \pm 0.27$ & $0.67 \pm 0.03$ & 395 \\
\hline
\end{tabular}

1) Weighted fits of the bisector through $N$ pairs of $(\log X, \log Y)$, where $N$ is the number of independent points (Isobe et al. 1990; Nieten 2001). ${ }^{a}\left(\mathrm{~K} \mathrm{~km} \mathrm{~s}^{-1}\right) ;{ }^{b}\left(\mathrm{MJy} \mathrm{sr}^{-1}\right) ;{ }^{c}\left(10^{18}\right.$ at cm $\left.{ }^{-2}\right)$.

selection of points in correlations involving $\mathrm{CO}$ intensities; likewise, the noise in the H I map determined the selection of points in correlations involving intensities of $\mathrm{HI}$ or total gas. In spite of this difference, the selected pixels are largely the same; most pixels are located on the broad emission ring. To obtain sets of independent data points, i.e. a beam area overlap of $\leq 5 \%$, only pixels spaced by $\geq 1.67 \times$ the beamwidth were considered. The resulting correlations between $\mathrm{CO}, \mathrm{HI}$ and the FIR are listed in Table 2, and examples of correlation plots are shown in Fig. 15.

In view of the distinct differences between the $\mathrm{CO}$ and $\mathrm{HI}$ distributions, it is not surprising that their velocity-integrated intensities are not well correlated (see Figs. 15a and 15b). In the panel showing the full radial range, $R=0^{\prime}-60^{\prime}$, two maxima occur that correspond to the inner bright $\mathrm{CO}$ arm and the main emission ring, respectively. In the inner arm the molecular fraction is much larger than in the main ring, and the decrease of this fraction outwards obviously contributes to the large spread in the points. Therefore we also analysed the inner $\left(R=0^{\prime}-35^{\prime}\right)$ and outer $\left(R=35^{\prime}-60^{\prime}\right)$ radial ranges separately. In the inner part $I_{1-0}$ and $I_{\mathrm{HI}}$ are not correlated (the correlation coefficient $\rho=0.3$, see Table 2), but for the outer range some correlation is visible (Fig. 15b), with $\rho=0.61 \pm 0.09$. Note that the correlation follows a power law with exponent $1.7 \pm 1.0$. An exponent of 2 is expected if the formation and destruction rates of $\mathrm{H}_{2}$ are balanced (Reach \& Boulanger 1998). This occurs in the denser H I phase at temperatures near $80 \mathrm{~K}$, which are also found in M 31 (Braun \& Walterbos 1992). For a better check of this possibility in M 31, this exponent could be determined in narrow radial ranges at the original resolution of $24^{\prime \prime} \times 36^{\prime \prime}$ of the H I map, which may yield better correlations than obtained here.

The middle panels of Fig. 15 show that in the interval $R=$ $35^{\prime}-60^{\prime}$ both $I_{\mathrm{HI}}$ and $I_{1-0}$ are well correlated with the dust emission at $175 \mu \mathrm{m}$. With correlation coefficients of $\rho=0.75 \pm 0.03$ and $\rho=0.81 \pm 0.02$, respectively, both correlations are highly significant (Table 2). The relationship between $I_{\mathrm{HI}}$ and $I_{175}$ is nearly linear, whereas that between $I_{1-0}$ and $I_{175}$ follows a power law with exponent $1.6 \pm 0.2$ (see Table 2). This difference reflects the power-law dependence of $I_{1-0}$ on $I_{\mathrm{HI}}$ in Fig. 15b. The two branches visible at $\log I_{175}<1.3$ in Fig. $15 \mathrm{c}$ are caused by the strong radial gradient in the apparent atomic gas-to-dust ratio $N(\mathrm{H} \mathrm{I}) / I_{175}$ plotted in Fig. 13: points in the upper branch are at larger radius than points in the lower one. This branching does 
not occur in the correlation between $I_{1-0}$ and $I_{175}$ (Fig. 15d), because $2 N\left(\mathrm{H}_{2}\right) / I_{175}$ increases much less with $R$ than $N(\mathrm{H} \mathrm{I}) / I_{175}$.

Correlations between the total gas column density and the $175 \mu \mathrm{m}$ emission for both the inner and the outer part of M 31 are better than those between each of the gas components alone and $I_{175}$ (see Table 2 and Figs. 15e and $\mathrm{f}$ ). The correlation for the outer part is very good $(\rho=0.85 \pm 0.02)$, much better than for the inner part $(\rho=0.68 \pm 0.05)$. Because in the inner part $N_{\text {gas }}$ is dominated by the molecular gas, but in the outer part by the atomic gas, the power-law exponent in the inner part (1.42 \pm 0.53$)$ is larger than in the outer part $(1.12 \pm 0.14)$. The latter exponent is consistent with a linear correlation between $N_{\text {gas }}$ and $I_{175}$. Linear correlations between gas and dust in M 31 were also found by other authors. Pagani et al. (1999) found a linear dependence of the intensity in the ISOCAM LW2 filter $(5.0-8.0 \mu \mathrm{m})$ on $N_{\text {gas }}$ in a field centred on the southern major axis near $X=-30^{\prime}$. In a spiral-arm region centred near $X=-20^{\prime}, Y=-7^{\prime}$ in the SW quadrant, Neininger et al. (1998) obtained a good linear correlation between $N_{\text {gas }}$ and the apparent red opacity. In the Milky Way, gas and dust were found to correlate in detail (Bohlin et al. 1978; Boulanger \& Pérault 1988; Boulanger et al. 1996).

One may ask why the $175 \mu \mathrm{m}$ dust emission follows $N_{\text {gas }}$ more closely than $N(\mathrm{HI})$ alone, since the gas is mainly atomic (see Table 2; see also Buat et al. 1989). There are several reasons for this. First, emission from dust in H I clouds, as well as from dust in $\mathrm{H}_{2}$ clouds, contributes to the $175 \mu \mathrm{m}$ emission. Second, according to dust models (see e.g. Mezger et al. 1990), the dust absorption coefficient and the dust emissivity per $\mathrm{H}$-atom are two (or more) times larger in the dense molecular clouds than in the diffuse atomic clouds. Hence, for equal dust column densities and temperatures, the emission from dust in $\mathrm{CO}$ clouds exceeds that from dust in $\mathrm{H}$ I clouds as soon as $2 N\left(\mathrm{H}_{2}\right) / N(\mathrm{HI})>0.3$.

The data presented in Sects. 4.1 and 4.2 show that a radial gradient in the apparent gas-to-dust ratio exists that influences the correlations between the gas components and the dust. Corrections for $\mathrm{H}$ I-line opacity, better knowledge of the conversion factor $X_{\mathrm{CO}}$ and, probably most important, a determination of the dust temperature as a function of radius are needed to understand whether this radial gradient reflects a real increase in the dust-to-gas ratio in M31. In the meantime comparisons of gas and dust on small scales in narrow radial ranges would be of interest.

\subsection{Molecular and total gas mass}

The CO intensity integrated over the area of M31 gives an estimate of the total molecular mass if one assumes a conversion factor between $I_{1-0}$ and $N\left(\mathrm{H}_{2}\right)$. We integrated the $\mathrm{CO}$ intensities in Fig. 1a out to a radius of $80^{\prime}$ in the plane of $\mathrm{M} 31$, which corresponds to $R=18 \mathrm{kpc}$. With the same conversion factor $X_{\mathrm{CO}}$ as used above we find a molecular mass of $M\left(\mathrm{H}_{2}\right)=3.45 \times 10^{8} M_{\odot}$. Along the major axis our map only extends to $R \simeq 55^{\prime}$, so we missed the emission near the major axis between $R=55^{\prime}$ and 75' detected by Koper et al. (1991). From Figs. 3b and 10c in Dame et al. (1993) we estimate that this contributes about $5 \%$ of the total. After correcting for these $5 \%$, we get $M\left(\mathrm{H}_{2}\right)=3.6 \times 10^{8} M_{\odot}$ within a radius $R=18 \mathrm{kpc}$. This is in excellent agreement with the value obtained by Dame et al. (1993), which is $M\left(\mathrm{H}_{2}\right)=$ $3.45 \times 10^{8} M_{\odot}$ after scaling it to the distance of $780 \mathrm{kpc}$ adopted here. Given that Dame et al. used the same value for $X_{\mathrm{CO}}$ as we do, we can also conclude that their CO intensity scale of radiation temperature, $T_{\mathrm{R}}$, closely agrees with our scale of main beam brightness temperature, $T_{\mathrm{mb}}$.
To obtain the atomic gas mass within $R=18 \mathrm{kpc}$, we integrated the Westerbork H I map of Brinks \& Shane (1984) giving $M(\mathrm{HI})=2.6 \times 10^{9} M_{\odot}$ in the optically thin case. Thus for $R<18 \mathrm{kpc}$, the $\mathrm{H}_{2}$ mass is $14 \%$ of the $\mathrm{HI}$ mass and $12 \%$ of the neutral gas mass. The total H I mass of M31 at the distance of $780 \mathrm{kpc}$ is $4.86 \times 10^{9} M_{\odot}$ (Cram et al. 1980, corrected by Dame et al. 1993), which gives a total neutral gas mass in M 31 of $M_{\text {gas }}=5.2 \times 10^{9} M_{\odot}$ and a molecular mass fraction $M\left(\mathrm{H}_{2}\right) / M_{\text {gas }}=0.07$.

The bulk of the dust in M 31 is cold dust at a temperature of $T_{\mathrm{d}}=16 \mathrm{~K}$. Haas et al. (1998) estimated a total dust mass of $M_{\mathrm{d}}=3.8 \times 10^{7} M_{\odot}$ at $D=780 \mathrm{kpc}$. However, Schmidtobreick et al. (2000) found $M_{\mathrm{d}}=1.3 \times 10^{7} M_{\odot}$ using the same data but a different method of calculation. Hence the apparent gas-to-dust mass ratio in M 31 is $M_{\text {gas }} / M_{\mathrm{d}}=137-410$. If the H I masses are corrected for opacity of the HI, they increase by $19 \%$ (Braun $\&$ Walterbos 1992) and the ratio becomes $M_{\text {gas }} / M_{\mathrm{d}}=163-488$. For the Milky Way, Sodroski et al. (1994) obtained a gas-to-dust mass ratio for the entire Galaxy of $M_{\mathrm{gas}} / M_{\mathrm{d}}=160 \pm 60$, similar to the lower estimates for M 31 .

\section{The CO velocity field}

Figure $1 \mathrm{~b}$ shows the first moment of the $\mathrm{CO}$ spectral data cube, i.e. the intensity-weighted mean $\mathrm{CO}$ velocity as a function of position. This figure can be compared with Fig. 15a of Brinks \& Burton (1986 - hereafter B\&B), which shows the first moment of the $\mathrm{HI}$ data cube. In contrast to $\mathrm{HI}$, CO emission becomes very weak at galactocentric radii $R>18 \mathrm{kpc}$, where the $\mathrm{H}$ I disk starts to flare and to warp (scaled to the distance of M 31 of $0.78 \mathrm{Mpc}$ ). The velocity field in Fig. $1 \mathrm{~b}$ is therefore little affected by foreground and background emission from the warp and is more appropriate to deriving the velocity field in the disk than the velocity field of HI. Moreover, as M31 is at a galactic latitude of $b=-22^{\circ}$, contamination by foreground Milky Way clouds at the velocities of interest here $\left(<-50 \mathrm{~km} \mathrm{~s}^{-1}\right)$ is unlikely. The average deviation between the $\mathrm{H} \mathrm{I}$ and $\mathrm{CO}$ mean velocities is only $\simeq 10 \mathrm{~km} \mathrm{~s}^{-1}$, which may imply that the bulk of the H I emission in the region covered by our CO map arises from M31's disk, although the different velocity resolutions $\left(8.2 \mathrm{~km} \mathrm{~s}^{-1}\right.$ for $\mathrm{HI}$ and $2.6 \mathrm{~km} \mathrm{~s}^{-1}$ for $\mathrm{CO}$ ) may contribute to the differences.

The distribution of the mean $\mathrm{CO}$ velocities is dominated by the rotation around the dynamical centre of the galaxy that has a velocity $V_{\text {sys }}=-315 \mathrm{~km} \mathrm{~s}^{-1}$. Only one small cloud near the centre of the galaxy and a large cloud complex near the northwestern minor axis show strong deviations from circular rotation. The small cloud is located at $X, Y=-0.1,3 ! 1\left(-250 \mathrm{~km} \mathrm{~s}^{-1}\right)$. Its non-circular orbit may be caused by the central bar (Berman 2001; Berman \& Loinard 2002).

The cloud complex near the minor axis $\left(X, Y=3^{\prime},-4^{\prime}\right)$, which appears particularly bright at $175 \mu \mathrm{m}$, shows complex line profiles that may be related to the complex filamentary structure visible in Fig. 7. Alternatively, they may trace streaming motions (Muller et al., in prep.).

The width of the observed CO-line profiles varies strongly from position to position. The average linewidth (second moment of the $\mathrm{CO}$ data cube) computed over the whole disk is $10 \pm 5 \mathrm{~km} \mathrm{~s}^{-1}$; it is higher in the arms $\left(15 \pm 5 \mathrm{~km} \mathrm{~s}^{-1}\right)$ and lower at the arm edges and in the interarm regions $\left(8 \pm 4 \mathrm{~km} \mathrm{~s}^{-1}\right)$. The average $\mathrm{CO}$ linewidth agrees with the average linewidth of the $\mathrm{H} \mathrm{I}$ emission of $8.1 \mathrm{~km} \mathrm{~s}^{-1}$ (at $1^{\prime}$ resolution) arising from the disk (Unwin 1983).

Some of the line profiles are very broad $\left(40-65 \mathrm{~km} \mathrm{~s}^{-1}\right.$, e.g. Fig. 2c) and show multiple velocity components. This is 


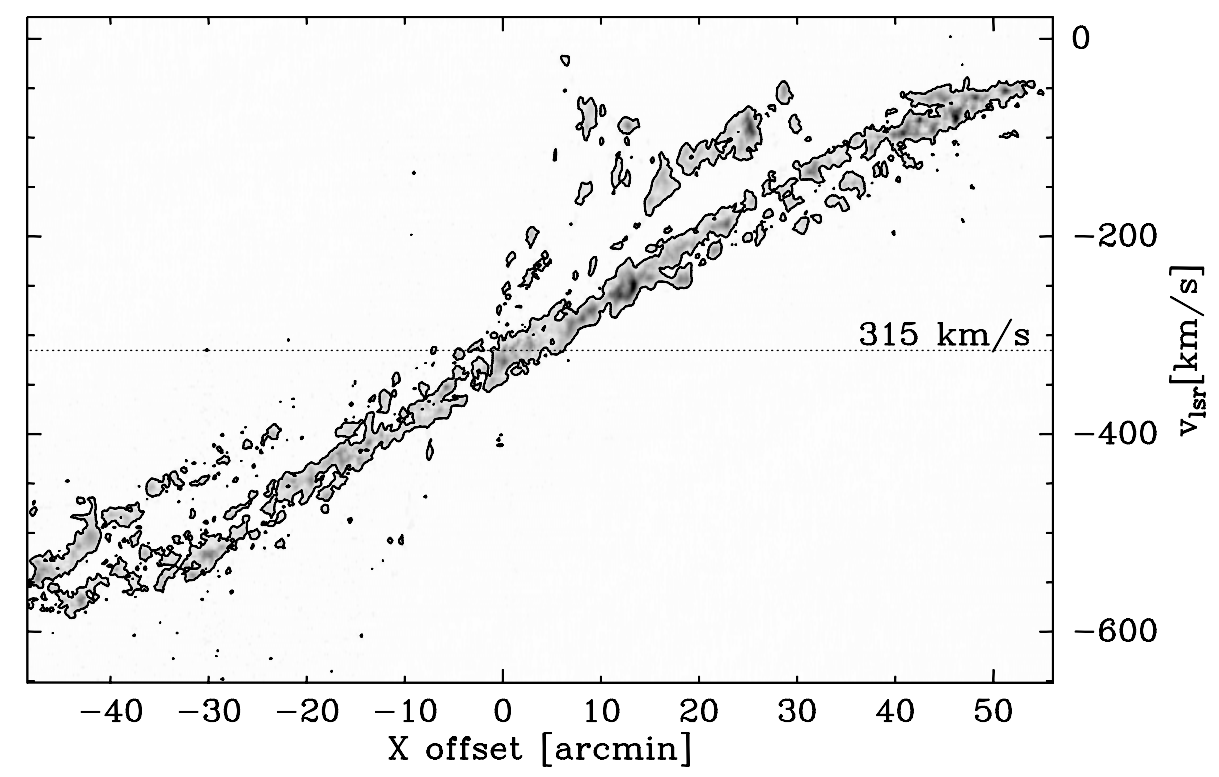

Fig. 16. Position-velocity diagram along the major axis obtained by adding the intensities along lines parallel to the $Y$-axis as function of position on the $X$-axis and velocity. The grey scale represents the intensities starting at contour level $0.5 \mathrm{~K}$. The dotted line indicates the system velocity of $-315 \mathrm{~km} \mathrm{~s}^{-1}$.

particularly the case for the cloud complexes associated with the dark clouds D 47 and D 39 ( $X, Y=-22.48,-7.53$ and $-41^{\prime} .9,8.54$, see Figs. $2 \mathrm{~g}$ and $2 \mathrm{i}$ ), which show complex profiles with total widths of 50 and $65 \mathrm{~km} \mathrm{~s}^{-1}$, respectively. We note that in the case of D 47 and D 39 the multiple profiles arise in the vicinity of bright $\mathrm{H}$ II regions, but this possible connection is not a general feature.

Other line profiles appear to be very narrow $\left(\leq 5 \mathrm{~km} \mathrm{~s}^{-1}\right.$; see Fig. 2b). The profiles associated with $\mathrm{D} 153(X, Y=$ -17 ' $8,-3$ ' 85 , see Fig. 2 f) have a particularly high peak intensity combined with a narrow line width of only about $4 \mathrm{~km} \mathrm{~s}^{-1}$.

A detailed analysis of the cloud-to-cloud velocity dispersions inside the molecular cloud complexes and of the interplay between density-wave driven motions and peculiar motions linked to H II regions or supernova remnants, will be the subject of a forthcoming paper (Muller et al., in prep.).

The position-velocity diagram of the CO (1-0) intensity integrated along the minor axis is shown in Fig. 16. The dominant features directly reflect the general velocity field in the disk (Fig. 1b) and the fact that most CO emission is concentrated in spiral arms (Figs. 1a, 3a). Due to the rotation of the M 31 disk, CO emission from the arms marked in Fig. 3a is seen in a broad band of about $100 \mathrm{~km} \mathrm{~s}^{-1}$ width, stretching from $X \simeq-48^{\prime}, v \sim-550 \mathrm{~km} \mathrm{~s}^{-1}$ to $X \simeq+55^{\prime}, v=-50 \mathrm{~km} \mathrm{~s}^{-1}$. Most of the emission in this band is from regions near $R=10 \mathrm{kpc}$. This large-scale picture agrees, of course, with that in the lowresolution survey by Dame et al. (1993), who also find some emission near $X=-63, v=-550 \mathrm{~km} \mathrm{~s}^{-1}$, outside the area of our survey. The $\mathrm{CO}$ spiral arms appear as loops within the range of this band (compare Braun (1991) for H I); most clearly seen in Fig. 16 is the loop at the most negative $X$ corresponding to the "dashed" spiral arm in Fig. 3a. Emission from the inner part of the "solid" spiral arm in Fig. 3a is clearly visible at $X<25^{\prime}, v \gtrsim-200 \mathrm{~km} \mathrm{~s}^{-1}$ above the main band of emission. Extended and relatively strong emission at velocities around $-80 \mathrm{~km} \mathrm{~s}^{-1}$ and $X$ about $8^{\prime}$ to $12^{\prime}$ is from clouds located inside the "solid" spiral arm in Fig. 3a, i.e. closer to the major axis (compare Fig. 1b). While we cannot reliably trace spiral arms from the $\mathrm{CO}$ emission in this inner region, we note that these $\mathrm{CO}$ features agree in position and velocity with the inner loops of the spiral arms identified by Braun (1991) from H I surveys. In a similar fashion, most smaller and weaker features at velocities that are different from those of the main CO spiral arms in Figs. $1 \mathrm{~b}$ and 3a are on other loops of Braun's H I spiral arms.

\section{Summary}

The new ${ }^{12} \mathrm{CO}(J=1-0)$-line survey of the Andromeda galaxy presented here covers an area of about $2^{\circ} \times 0.5$, which is fully sampled with a velocity resolution of $2.6 \mathrm{~km} \mathrm{~s}^{-1}$ and an angular resolution of $23^{\prime \prime}$, the highest angular resolution to date of a map of this extent. At the adopted distance of $780 \mathrm{kpc}$ the linear resolution is $85 \mathrm{pc} \times 400 \mathrm{pc}$ in the plane of M31. The On-the-Fly method of observing made it possible to measure nearly 1.7 million spectra (before gridding) in about $500 \mathrm{~h}$ of effective observing time on $\mathrm{M} 31$. The rms noise in the $\mathrm{CO}(1-0)$ line per $1 \mathrm{MHz}$ channel is $25 \mathrm{mK}$ in the northern half of the map and $33 \mathrm{mK}$ in the southern half.

The velocity-integrated distribution, $I_{1-0}$, and the velocity field are shown in Fig. 1. The molecular gas is concentrated in narrow, filamentary arms between 4 and $12 \mathrm{kpc}$ from the centre with a maximum near $10 \mathrm{kpc}$. The inner arm at $R \simeq 5 \mathrm{kpc}$ is remarkably bright. Only a few clouds, often forming bridge-like structures, were detected between the arms above $3 \times$ rms noise of typically $0.35 \mathrm{~K} \mathrm{~km} \mathrm{~s}^{-1}$. The region within $2 \mathrm{kpc}$ from the centre is almost free of molecular clouds that are brighter than the sensitivity of this survey.

The thin CO arms define a two-armed spiral pattern that can be described well by two logarithmic spirals with a constant pitch angle of $7^{\circ}-8^{\circ}$. At a resolution of $45^{\prime \prime}$, the arm-interarm contrast reaches a maximum of 20 in $I_{1-0}$ compared to 4 in $\mathrm{H} \mathrm{I}$ for the western bright arms. The H I arms are much wider than the molecular arms, and diffuse H I exists everywhere between the arms and at large radii. Few molecular clouds are visible outside a radius of $16 \mathrm{kpc}$. 
The velocity field of the molecular gas is very similar to that of the disk component in H I. At some positions perturbed velocity profiles occur that are possibly caused by nearby H II regions.

Several selected regions were also observed in the ${ }^{12} \mathrm{CO}(2-1)$ line. At a resolution of $23^{\prime \prime}$, the line ratios are nearly constant with mean values of $I_{2-1} / I_{1-0}=0.5-0.7$ in the arms. These line ratios are similar to those observed in other galaxies and show no indication of subthermal excitation.

Averaged radial profiles of the velocity-integrated $\mathrm{CO}$ and H I distributions show that, for a constant conversion factor of $X_{\mathrm{CO}}=1.9 \times 10^{20} \mathrm{~mol} \mathrm{~cm} \mathrm{~cm}^{-2}\left(\mathrm{~K} \mathrm{~km} \mathrm{~s}^{-1}\right)^{-1}$, the molecular fraction of the neutral gas is enhanced on the spiral arms and decreases radially from about 0.6 on the inner arms to about 0.3 on the arms at $R \simeq 10 \mathrm{kpc}$ (see Fig. 11). The molecular fraction also varies considerably along the arms. Comparisons of the $\mathrm{H} \mathrm{I}$ and $\mathrm{CO}$ profiles with the averaged radial profile of the $\lambda 175 \mu \mathrm{m}$ emission from cold $(16 \mathrm{~K})$ dust revealed a strong, continuous increase of the apparent atomic gas-to-dust ratio of nearly a factor 20 between the centre and $R \simeq 14 \mathrm{kpc}$, whereas the apparent molecular gas-to-dust ratio increases by about a factor of 4 . The apparent total gas-to-dust ratio also increases by about a factor of 20 .

The strong apparent gradients in the molecular fraction and the gas-to-dust ratios influence the cross-correlations between $\mathrm{CO}, \mathrm{HI}$ and FIR $(175 \mu \mathrm{m})$ intensities. In the radial range $R=$ $35^{\prime}-60^{\prime}$ (about 8-14 kpc), the best correlation exists between total neutral gas and $175 \mu \mathrm{m}$, followed by the correlations between $\mathrm{CO}$ and $175 \mu \mathrm{m}$ and between $\mathrm{HI}$ and $175 \mu \mathrm{m}$. The relationships between H I and $175 \mu \mathrm{m}$ and between the total gas and $175 \mu \mathrm{m}$ are close to linear, whereas that between $\mathrm{CO}$ and $175 \mu \mathrm{m}$ is a power law with exponent 1.6 due to a possible non-linearity between $\mathrm{CO}$ and H I. In the inner part of M31, $R<35^{\prime}$ (but outside the nuclear area), only total gas and FIR $(175 \mu \mathrm{m})$ are reasonably well correlated (see Table 2).

The total molecular mass of M 31 within a radius of $18 \mathrm{kpc}$ is $3.6 \times 10^{8} M_{\odot}$, when using the above-mentioned value of $X_{\mathrm{CO}}$. As the total H I mass (without correction for opacity) is $4.86 \times$ $10^{9} M_{\odot}$, the total mass of the neutral gas is $5.2 \times 10^{9} M_{\odot}$. The total mass of the cold dust is $(1.3-3.8) \times 10^{7} M_{\odot}$, so the total gas-to-dust mass ratio is 410-137. The lower value agrees with the Galactic one.

The wealth of information contained in our new ${ }^{12} \mathrm{CO}(1-0)$ survey of M 31 enables a number of new investigations into the physical relationships between molecular gas, atomic hydrogen gas, cold and warm dust, ionized gas, relativistic electrons and magnetic fields. Such studies will be the subject of forthcoming papers.

The data shown in Fig. 1 can be obtained from M. Guélin (guelin@iram.fr).

Acknowledgements. C.N. acknowledges support from the Studienstiftung des Deutschen Volkes. We thank W. Brunswig, C. Kramer, G. Paubert, J. Schraml and A. Sievers for their important contributions to the On-the-Fly observing mode at the IRAM 30-m telescope and P. Hoernes for observations and software adjustment. S. Muller kindly communicated to us some results of his Ph.D. Thesis prior to publication. We thank M.-C. Willaime for sending us an updated version of the ISOCAM LW6 map that we used in Fig. 7. The extensive comments and suggestions of an anonymous referee were a great help in improving the manuscript. This work was started when M.G. was visiting the MPIfR as a von Humboldt Fellow.

\section{References}

Allen, R. J., \& Lequeux, J. 1993, ApJ, 410, L15

Baade, W. 1963, in Evolution of stars and galaxies, ed. C. Payne-Gaposhkin (Cambridge: Harvard University Press), 56
Baade, W., \& Arp, H. 1964, ApJ, 139, 1027

Bajaja, E., \& Gergely, T. E. 1977, A\&A, 61, 229

Beck, R., Berkhuijsen, E. M., \& Hoernes, P. 1998, A\&AS, 129, 329

Berkhuijsen, E. M. 1997, in The Physics of Galactic Halos, Proc. 156th WE-

Heraeus Seminar, ed. H. Lesch, R.-J. Dettmar, U. Mebold, \& R. Schlickeiser (Berlin: Akademie Verlag), 155

Berkhuijsen, E. M., Bajaja, E., \& Beck, R. 1993, A\&A, 279, 359

Berkhuijsen, E. M., Nieten, Ch., \& Haas, M. 2000, in The Interstellar Medium in M 31 and M33, Proc. 232th WE-Heraeus Seminar, ed. E. M. Berkhuijsen, R. Beck, \& R. A. M. Walterbos (Aachen: Shaker), 187

Berman, S. 2001, A\&A, 371, 476

Berman, S., \& Loinard, L. 2002, MNRAS, 336, 477

Bohlin, R. C., Savage, B. D., \& Drake, J. F. 1978, ApJ, 224, 132

Boulanger, F., \& Pérault, M. 1988, ApJ, 330, 964

Boulanger, F., Abergel, A., \& Bernard, J.-P. 1996, A\&A, 312, 256

Braine, J., Combes, F., Casoli, F., et al. 1993, A\&AS, 97, 887

Braun, R. 1990, ApJS, 72, 762

Braun, R. 1991, ApJ, 372, 54

Braun, R., \& Walterbos, R. A. M. 1992, ApJ, 386, 120

Brinks, E., \& Shane, W. W. 1984, A\&AS, 55, 179 [B\&S]

Brinks, E., \& Burton, W. B. 1986, A\&A, 141, 195

Buat, V., Deharveng, J. M., \& Donas, J. 1989, A\&A, 223, 42

Cram, T. R., Roberts, M. S., \& Whitehurst, R. N. 1980, A\&AS, 40, 215

Dame, T. M., Koper, E., Israel, F. P., et al. 1993, ApJ, 418, 730

Dame, T. M., Hartmann, D., \& Thaddeus, P. 2001, ApJ, 547, 792

Downes, D. 1989, in Evolution of Galaxies, Astronomical Observations, ed. I. Appenzeller, H. J. Habing, \& P. Lena (Berlin: Springer), 351

Dressel, L. L., \& Condon, J. J. 1976, ApJS, 31, 187

Emerson, D. T. 1974, MNRAS, 169, 607

Emerson, D. T., \& Gräve, R. 1988, A\&A, 190, 353

Engargiola, G., Plambeck, R. L., Rosolowsky, E., \& Blitz, L. 2003, ApJS, 149, 343

Garcia-Burillo, S., Guélin, M., Cernicharo, J., \& Dahlem, M. 1992, A\&A, 266, 21

Garcia-Burillo, S., Guélin, M., \& Cernicharo, J. 1993, A\&A, 274, 123

Gordon, K., Rieke, G., Krause, O., et al. 2004, A\&AS, 20514104G

Greve, A., Kramer, C., \& Wild, W. 1998, A\&AS, 133, 271

Guélin, M., Nieten, C., Neininger, N., et al. 2000, in The Interstellar Medium in M 31 and M33, Proc. 232th WE-Heraeus Seminar, ed. E. M. Berkhuijsen, R. Beck, \& R. A. M. Walterbos (Aachen: Shaker), 15

Guélin, M., Muller, S., Nieten, Ch., et al. 2004, in The Dense Interstellar Medium in Galaxies, ed. S. Pfalzner, C. Kramer, C. Straubmeier, \& A. Heithausen (Berlin: Springer), 121

Haas, M., Lemke, D., Stickel, M., et al. 1998, A\&A, 338, L33

Handa, T., Hasegawa, T., Morino, J. I., et al. 2002, in The Universe at Low Radio Frequencies, ed. A. Pramesh Rao, G. Swarup, \& Gopal-Krishna, IAU Symp., 199, 339

Heyer, M. H., Dame, T. M., \& Thaddeus, P. 2000, in The Interstellar Medium in M 31 and M33, Proc. 232th WE-Heraeus Seminar, ed. E. M. Berkhuijsen, R. Beck, \& R. A. M. Walterbos (Aachen: Shaker), 29

Heyer, M. H., Corbelli, E., Schneider, S. E., et al. 2004, ApJ, 602, 723

Hodge, P. L. 1979, AJ, 84, 744

Hodge, P. L. 1981a, Atlas of the Andromeda Galaxy (University of Washington Press)

Hodge, P. L. 1981b, Scient. Amer., 244, 92

Hoernes, P. 1997, Ph.D. Thesis, University of Bonn

Hoernes, P., Berkhuijsen, E. M., \& Xu, C. 1998, A\&A, 334, 57

Honma, M., Sofue, Y., \& Arimoto, N. 1995, A\&A, 304, 1

Hubble, E. 1929, ApJ, 69, 103

Isobe, T., Feigelson, E. D., Akritas, M. G., \& Babu, G. J. 1990, ApJ, 364, 104

Israel, F. P., Johansson, L. E. B., Lequeux, J., et al. 1993, A\&A, 276, 25

Israel, F. P., Tilanus, R. P. J., \& Baas, F. 1998, A\&A, 339, 398

Keel, W. C. 2000, in The Interstellar Medium in M 31 and M 33, Proc. 232th WEHeraeus Seminar, ed. E. M. Berkhuijsen, R. Beck, \& R. A. M. Walterbos (Aachen: Shaker), 159

Koper, E., Israel, F. P., Dame, T. M., \& Thaddeus, P. 1991, ApJ, 383, L11

Lequeux, J. 2000, in The Interstellar Medium in M 31 and M33, Proc. 232th WE-Heraeus Seminar, ed. E. M. Berkhuijsen, R. Beck, \& R. A. M. Walterbos (Aachen: Shaker), 63

Loinard, L., \& Allen, R. J. 1998, ApJ, 499, 227

Loinard, L., Allen, R. J., \& Lequeux, J. 1995, A\&A, 301, 68

Loinard, L., Dame, T. M., Koper, E., et al. 1996, ApJ, 469, L101

Loinard, L., Dame, T. M., Heyer, M. H., et al. 1999, A\&A, 351, 1087

Melchior, A.-L., Viallefond, F., Guélin, M., \& Neininger, N. 2000, MNRAS, 312, L29

Mezger, P. G., Zylka, R., \& Wink, J. E. 1990, A\&A, 228, 95

Moshir, M., Marsh, K., Price, S., \& Shipman, R. 1999, in Astrophysics with Infrared Surveys: A Prelude to SIRTF, ed. M. D. Bicay, R. M. Cutri, \& B. F. 
Madore, ASP Conf. Ser., 177, 212

Moss, D., Shukurov, A., Sokoloff, D. D., et al. 1998, A\&A, 335, 500

Muller, S. 2003, Ph.D. Thesis, University Joseph Fourier, Grenoble

Nakano, M., Ichikawa, T., Tanaka, Y., et al. 1987, PASJ, 39, 57

Nedialkov, P., Berkhuijsen, E. M., Nieten, C., et al. 2000, in The Interstellar Medium in M31 and M33, Proc. 232th WE-Heraeus Seminar, ed. E. M. Berkhuijsen, R. Beck, \& R. A. M. Walterbos (Aachen: Shaker), 85

Neininger, N., Guélin, M., Ungerechts, H., Lucas, R., \& Wielebinski, R. 1998, Nature, 395, 871

Neininger, N., Guélin, M., Lucas, R., \& Muller, S. 2000a, in The Interstellar Medium in M 31 and M 33, Proc. 232th WE-Heraeus Seminar, ed. E. M. Berkhuijsen, R. Beck, \& R. A. M. Walterbos (Aachen: Shaker), 25

Neininger, N., Nieten, Ch., Wielebinski, R., et al. 2000b, in Imaging at Radio through Submillimeter Wavelengths, ed. J. G. Mangum, \& S. J. E. Radford, ASP Conf. Ser., 217, 72

Nieten, Ch. 2001, Ph.D. Thesis, University of Bonn

Nieten, Ch., Neininger, N., Guélin, M., et al. 2000, in The Interstellar Medium in M 31 and M 33, Proc. 232th WE-Heraeus Seminar, ed. E. M. Berkhuijsen, R. Beck, \& R. A. M. Walterbos (Aachen: Shaker), 21

Pagani, L., Lequeux, J., Cesarsky, D., et al. 1999, A\&A, 351, 447

Reach, W. T., \& Boulanger, F. 1998, in The Local Bubble and Beyond, ed. D. Breitschwerdt, M. J. Freyberg, \& J. Trümper (Berlin: Springer), 353

Regan, M. W., Thornley, M. D., Helfer, T. T., et al. 2001, ApJ, 561, 218
Ryden, B. S. \& Stark, A. A. 1986, ApJ, 305, 823

Sakamoto, S., Hasegawa, T., Handa, T., et al. 1997, ApJ, 486, 276 Savcheva, A., \& Tassev, S. 2002, Publ. Astron. Obs. Belgrade, 73, 219 Schmidtobreick, L., Haas, M., \& Lemke, D. 2000, A\&A, 363, 917 Sodroski, T. J., Bennett, C., Boggess, N., et al. 1994, ApJ, 420, 638 Sodroski, T. J., Odegard, N., Dwek, E., et al. 1995, ApJ, 452, 262 Stanek, K. Z., \& Garnavich, P. M. 1998, ApJ, 503, L131

Stark, A. A., \& Binney, J. 1994, ApJ, 426, L31

Strong, A. W., \& Mattox, J. 1996, A\&A, 308, L21

Strong, A. W., Moskalenko, I. V., Reimer, O., et al. 2004, A\&A, 422, L47

Unwin, S. C. 1983 , MNRAS, 205, 773

Walterbos, R. A. M. 2000, in The Interstellar Medium in M 31 and M33, Proc. 232th WE-Heraeus Seminar, ed. E. M. Berkhuijsen, R. Beck, \& R. A. M. Walterbos (Aachen: Shaker), 99

Walterbos, R. A. M., \& Kennicutt, R. C. 1988, A\&A, 198, 61

Willaime, M.-C., Lequeux, J., Melchior, A.-L., \& Hanus, M. 2001, in The Promise of the Herschel Space Observatory, ed. G. L. Pilbratt et al., ESA SP-460, 519

Williams, J. P., de Geus, E. J., \& Blitz, L. 1994, ApJ, 428, 693

Wilson, C. D. 1995, ApJ, 448, L97

Xu, C., \& Helou, G. 1996, ApJ, 456, 163

Young, J. S., Xie, S., Tacconi, L., et al. 1995, ApJS, 98, 219 